\title{
Electrically conducting sandwich cylinder with a planar lattice core under prescribed eigenstrain and magnetic field
}

\author{
A.H.Akbarzadeh ${ }^{1,2 *}$, J.W.Fu ${ }^{3}$, L.Liu ${ }^{2}$, Z.T.Chen ${ }^{4}$, D.Pasini2 ${ }^{*}$ \\ ${ }^{1}$ Department of Bioresource Engineering, McGill University, Ste-Anne-de-Bellevue, Island of Montreal, QC, \\ H9X 3V9, Canada \\ ${ }^{2}$ Department of Mechanical Engineering, McGill University, Montreal, QC, H3A 0C3, Canada \\ ${ }^{3}$ Department of Mechanical Engineering, Nanjing University of Science and Technology, Nanjing, 210094, \\ China \\ ${ }^{4}$ Department of Mechanical Engineering, University of Alberta, Edmonton, AB, T6G 2G8, Canada
}

\begin{abstract}
The eigenstrain theory is widely used to study inelastic responses of several classes of materials subjected to phase transformation, thermal expansion, and other multiphysics excitations. In this paper, we focus on electrically conducting cellular solids and examine their magnetoelastic responses when used as a core of a sandwich cylinder subjected to an eigenstrain and an external magnetic field. The cylinder comprises layers of either solid or cellular material and undergoes either plane strain or plane stress conditions in both time-harmonic and transient states. We use direct homogenization techniques (standard mechanics and micromechanical models) along with Bessel, Struve, and Lommel functions to study the roles that cell topology, relative density, eigenstrain, and bonding interface play on the magnetoelastic responses of the sandwich cylinder. The results show that relative density, cell topology, and magnetic field are the factors that most contribute to control the sandwich response. We also show that a careful tailoring of relative density and cell topology can lead to the simultaneous weight and overall stress reduction with improved natural frequency.
\end{abstract}

Keywords: Cell topology, Eigenstrain, Homogenization, Magnetoelasticity, Periodic cellular material, Relative density, Sandwich cylinder.

\section{Introduction}

In a homogenous body free from external forces and surface constraints, inclusion refers to a finite subdomain subjected to a prescribed eigenstrain [1]. Thermal expansion, swelling strain, phase transformation, plastic deformation, and piezoelectric/piezomagnetic strain are examples of nonelastic strains, namely eigenstrains [2-4]. The eigenstrain theory enables in a

\footnotetext{
Address correspondence to: A.H.Akbarzadeh: hamid.akbarzadeh@mcgill.ca and D.Pasini: damiano.pasini@mcgill.ca.
} 
single mathematical formulation to study each of these phenomena, including the response of an electrically conducting material. It can also be used to study the impact of microstructural imperfections in homogeneous and heterogeneous media.

Several works in the literature resorted to the eigenstrain theory to study problems dealing with the three-dimensional nanostrain in Ni-Ti shape memory alloys [5], residual strain measurements [6], stress changes caused by local deboning and damage evolution [7], and strain in living or non-living tissues [8]. Early micromechanical investigations on the eigenstrain were pioneered by Eshelby [9] as well as Mura and Kinoshita [10]. Since then, the studies that followed mainly focused on the application of eigenstrain in particulate composites and residual stress measurements [11-22]. For example, Liang et al. [23] obtained the stress field induced by an eigenstrain within an ellipsoidal inclusion in a thin film of a microelectromechanical system. The effect of the thin film's thickness on the induced eigenstress was found to decrease with an increase in the film thickness. The problem of an arbitrary-shaped heterogeneity, undergoing an eigenfield in a uniform magnetoelectroelastic load, was also examined. Shen and Hung [24], for instance, observed that the selection of appropriate eigenfields could effectively reduce the eigenstress developed in piezoelectric and piezomagnetic composites.

Sandwich structures are routinely used in automotive, aerospace, and sports equipment, among other sectors [25]. In the literature, there exists a large body of research on this subject across the length scale spectrum. In general, previous works focus on the micromechanics and multiphysics responses, such as elastodynamic, thermoelastic, and torsional rigidity [26-28]. The core of a typical sandwich panel is commonly made of either foams, e.g. polyurethane and polyethylene foams, or periodic cellular solids, e.g. honeycombs, corrugated metals, and other lattices [29, 30]. Their cellular architecture is often preferred to their solid counterpart not only for their low mass, but also for their ability to satisfy multifunctional requirements, such as those prescribed for heat exchangers, piezoelectric transducers, and bone tissue scaffolds [31-33]. For example, thermal expansion, electric conductivity, and flow permeability are some of the properties controlled by the geometry of the unit cell, in particular its topology, nodal connectivity, and relative density. While there are many studies on the multifunctional properties of cellular materials [34-43], few of them have used an eigenstrain analysis to study their mechanical and multiphysic properties, and to investigate the residual stress within the sandwich structure. For example, Schjodt-Thomsen and Pyrz [44] used a model based on Eshelby's equivalent eigenstrain to analyze the influence of alternative statistical cell dispersions on the local strain and the overall effective properties. The model was capable of capturing the effect of microstructural morphology on the stiffness properties. Nguyen et al. [45] examined the homogenization problem by imposing an eigenstrain that represents the thermal or piezoelectric strain within the representative volume element (RVE). The authors highlighted the simplicity of their model which was then used to obtain the effective properties. Moreover, Liu and Liang [46], through a micromechanics approach, obtained the effective elastic moduli of triangular lattices with microstructural defects. In this case, the use of eigenstrain was proved to be effective in the assessment of the role of defects occurring during the manufacturing of a set of cellular solids. 
More recently, electrically conducting cellular structures under a prescribed magnetic field have garnered a great deal of attention in a range of applications, such as medical microrobots driven by magnetic actuations [47], hydrophones [48], and metamaterials with negative permeability and negative refraction properties for optics [49]. Advances in additive manufacturing have also enabled the fabrication of cellular solids with electrically conducting microarchitecture, specifically for real-time measurement of structural performance [50-52]. Sandwich structures with electrically conducting solids experience the Lorentz force, according to the uncoupled magnetoelasticity theory [53], which dictates their multifunctional responses. To date, however, the behaviour of a sandwich cylinder with an electrically conducting cellular core subjected to a prescribed magnetic field has been rarely studied in the literature. To the best knowledge of authors, the analysis conducted in reference [37] is the only contribution considering the effect of magnetic field on the behavior of porous sandwich structures where the cellular layers have square cells. This subject matter is thus the focus of this work, which contributes to the body of literature dealing with the multiphysics analysis of cellular materials in the presence of eigenstrain.

The following clarifies the differences and originality that distinguish this work from that in reference [37]. This paper focuses on the role of cell topology in the magnetic properties of a periodic cellular solid. Besides the square cell, additional four cell topologies are investigated for the first time in this paper. For each of them, we present closed-form expressions describing the role of cell topology in the response of a sandwich cylinder subjected to a non-uniform eigenstrain which can assume any arbitrary form. Reference [37], on the other hand, studies the effect of relative density and property gradients for cellular layers with square cells. In addition, this work studies the effect of bonding imperfections on the multiphysics responses of lightweight sandwich cylinders, as opposed to our previous work which assumes perfect bonding among layers. Furthermore, here we present expressions for the effective magnetic permeability that are obtained numerically via standard mechanics and theoretically with closed form bounds. We also conduct a finite element analysis to validate the theoretical predictions. In reference [37], the relative density is assumed to vary linearly and only the Voigt model is used for the calculation; the results presented in this paper show how crude those approximations are, with inaccuracy up to $75 \%$ for the effective magnetic permeability.

The paper is organized as follows. Selected planar topologies for the unit cell are examined in section 2 and their effective magnetoelastic properties are obtained via standard mechanics homogenization. In sections 3 and 4, a dynamic eigenstrain excitation in the radial direction is expressed as a polynomial with an arbitrary order, which can be applied to any layer of a sandwich cylinder. The results are verified with finite element results and those found in the literature (Sections 5.1 and 5.2). The last part of the paper studies the influence of bonding imperfection, eigenstrain distribution, external magnetic field, cell topology, and relative density, besides mapping these factors in charts that illustrate the time-harmonic responses of alternative sandwich layouts.

\section{Effective magnetoelastic properties of periodic cellular solids}


Homogenization theory is commonly used to determine the effective properties of cellular solids and other periodic materials [54-58]. Many methods have been proposed, including micropolar theory, standard mechanics, asymptotic homogenization, and micromechanical models $[36,59]$, and effectively used to predict the properties of a cellular domain from of a limited portion of it, namely the Representative Volume Element. One main advantage of homogenization methods is the reduced computation cost as compared to a fully detailed analysis, where each cell element would be individually modelled. In this section, we present the effective magnetoelastic properties obtained by standard mechanics (numerical homogenization) and micromechanical closed-form expressions.

For the cellular core of the cylinder, we examine five alternative planar topologies of the unit cell (Figs. 1 and 2): square, mixed (triangular) A, and mixed (triangular) B with cubic symmetry, and Kagome and triangular with isotropic properties. Their material properties are conveniently expressed as a function of their relative density $\rho_{r}$ :

$$
\rho_{r}=\frac{\bar{\rho}}{\rho_{s}}
$$

where $\bar{\rho}$ and $\rho_{s}$ are, respectively, the effective density of the unit cell of cellular solids and the density of the constituent solid material.

Standard mechanics homogenization with periodic boundary conditions applied to each unit cell (Figs. 1 and 2), along with the classical elasticity theory are here used to obtain the effective magnetoelastic properties numerically. In particular, the effective stiffness and magnetic permeability tensors of an electrically conductive representative volume element (RVE) are here expressed as:

$$
\bar{C}_{i j k l}=\frac{1}{V_{R V E}} \int C_{i j n n} M_{m n k l}^{C} d V_{R V E}, \bar{\mu}_{i j}=\frac{1}{V_{R V E}} \int \mu_{i k} M_{k j}^{\mu} d V_{R V E}
$$

where $C_{i j k l}$ and $\mu_{i j}(i, j, k, l, m, n=1,2,3)$ are stiffness and magnetic permeability tensors, $V_{R V E}$ represents the RVE volume (for a planar RVE, $V_{R V E}$ is replaced by the area $A_{R V E}$ ). Local structural $\left(M_{i j k l}^{C}\right)$ and local magnetic $\left(M_{i j}^{\mu}\right)$ tensors are defined as:

$$
\varepsilon_{i j}=M_{i j k l}^{C} \bar{\varepsilon}_{k l}, \varphi_{, i}=M_{i j}^{\mu} \bar{\varphi}_{, j}
$$

where $\varepsilon_{i j}$ and $\varphi$ represent strain tensor and magnetic potential, respectively [25]. The overbar in Eqs. (1) - (3) stands for the effective properties $\left(\bar{\rho}, \bar{C}_{i j k l}, \bar{\mu}_{i j}\right)$ or average fields $\left(\bar{\varepsilon}_{i j}, \bar{\varphi}\right)$. For planar lattices, the components of the local structural and local magnetic tensors are first evaluated by using Eq. (3) and by applying three independent unit strains and two independent unit magnetic field intensities (spatial derivatives of magnetic potential) [54]. Then, the effective magnetoelastic properties are obtained via Eq. (2). Herein, the numerical results have been fitted via a fourth-order polynomial that best reduces the curve-fitting error:

$$
\bar{\chi}\left(\rho_{r}\right)=P_{0}+P_{1} \rho_{r}+P_{2} \rho_{r}^{2}+P_{3} \rho_{r}^{3}+P_{4} \rho_{r}^{4}
$$


with $\bar{\chi}$ representing a given effective property, such as normalized Young's modulus $\left(\bar{E} / E_{s}\right)$, Poisson's ratio $\left(\bar{v} / v_{s}\right)$, shear modulus $\left(\bar{G} / G_{s}\right)$, and magnetic permeability $\left(\bar{\mu} / \mu_{s}\right)$. The subscript " $s$ " represents the properties of a given solid material. The coefficients $P_{i}(i=0,1,2,3,4)$, given in Tables 1 and 2 , pertain to the plane strain and plane stress conditions. In the following sections, we drop the overbar symbol for the effective properties of cellular materials to simplify the notation.

Table 1. Curve fitting coefficients for effective material properties of square cell under plane strain condition

\begin{tabular}{|c|c|c|c|c|c|c|}
\hline Cell Topology & $\begin{array}{c}\text { Effective } \\
\text { Properties }\end{array}$ & $P_{0}$ & $P_{1}$ & $P_{2}$ & $P_{3}$ & $P_{4}$ \\
\hline \multirow{4}{*}{ Square } & $\frac{\overline{\bar{E}}}{\overline{E_{s}}}$ & 0.0242 & 0.1544 & 1.7328 & -2.6485 & 1.7282 \\
\hline & $\frac{\bar{v}}{v_{s}}$ & -0.0336 & 1.2921 & -2.3185 & 3.7418 & -1.6554 \\
\hline & $\frac{\bar{G}}{E_{s}}$ & -0.0103 & 0.1275 & -0.4206 & 0.4226 & 0.2678 \\
\hline & $\frac{\bar{\mu}}{\mu_{s}}$ & 0.0105 & 0.4793 & 0.3522 & -0.3113 & 0.4691 \\
\hline
\end{tabular}

Table 2. Curve fitting coefficients for effective material properties for alternative $2 \mathrm{D}$ cell topologies under plane stress condition.

\begin{tabular}{|c|c|c|c|c|c|c|}
\hline Cell Topology & $\begin{array}{c}\text { Effective } \\
\text { Properties } \\
\end{array}$ & $P_{0}$ & $P_{1}$ & $P_{2}$ & $P_{3}$ & $P_{4}$ \\
\hline \multirow{4}{*}{ Square } & $\frac{\bar{E}}{E_{s}}$ & 0.0104 & 0.2868 & 1.3521 & -2.2736 & 1.6222 \\
\hline & $\frac{\bar{v}}{v_{s}}$ & 0.0104 & 0.7914 & -0.7211 & 1.6908 & -0.7467 \\
\hline & $\frac{\overline{\bar{G}}}{E_{s}}$ & 0.0032 & 0.0524 & -0.1853 & 0.01245 & 0.3884 \\
\hline & $\frac{\bar{\mu}}{\mu_{s}}$ & 0.0105 & 0.4793 & 0.3522 & -0.3113 & 0.4691 \\
\hline \multirow{2}{*}{ Kagome } & $\frac{\bar{E}}{E_{s}}$ & 0.0022 & 0.2875 & 0.4348 & -0.6018 & 0.8755 \\
\hline & $\frac{\bar{v}}{v_{s}}$ & 0.9944 & 0.1857 & -0.4195 & 0.1036 & 0.1386 \\
\hline
\end{tabular}


A.H. Akbarzadeh et al.

\begin{tabular}{|c|c|c|c|c|c|c|}
\hline 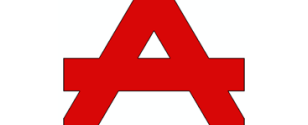 & $\frac{\bar{G}}{E_{s}}$ & 0.0005 & 0.1187 & 0.1112 & -0.1405 & 0.2843 \\
\hline & $\frac{\bar{\mu}}{\mu_{s}}$ & 0.0101 & 0.5074 & 0.0973 & 0.2941 & 0.09 \\
\hline \multirow[t]{4}{*}{ Triangular } & $\frac{\bar{E}}{E_{s}}$ & 0.0198 & -0.0608 & 2.2346 & -3.9441 & 2.7464 \\
\hline & $\frac{\bar{v}}{v_{s}}$ & 1.0186 & -0.1654 & 1.8532 & -4.2593 & 2.5492 \\
\hline & $\frac{\bar{G}}{E_{s}}$ & 0.0055 & 0.0155 & 0.6347 & -1.1232 & 0.8415 \\
\hline & $\frac{\bar{\mu}}{\mu_{s}}$ & 0.0129 & 0.4065 & 0.781 & -1.2302 & 1.0273 \\
\hline \multirow{4}{*}{ Mixed triangular A } & $\frac{\bar{E}}{E_{s}}$ & 0.021 & -0.1402 & 2.4344 & -4.3914 & 3.0714 \\
\hline & $\frac{\bar{v}}{v_{s}}$ & 1.2744 & -0.4365 & 3.2031 & -6.831 & 3.7828 \\
\hline & $\frac{\overline{\bar{G}}}{E_{s}}$ & 0.0066 & 0.0086 & 0.7849 & -1.421 & 0.9944 \\
\hline & $\frac{\bar{\mu}}{\mu_{s}}$ & 0.014 & 0.3728 & 0.9712 & -1.6004 & 1.2379 \\
\hline \multirow{4}{*}{ Mixed triangular B } & $\frac{\bar{E}}{E_{s}}$ & 0.0239 & -0.1083 & 2.6699 & -4.7654 & 3.1746 \\
\hline & $\frac{\bar{v}}{v_{s}}$ & 2.5257 & -4.4859 & 2.273 & -0.1233 & 0.8051 \\
\hline & $\frac{\bar{G}}{E_{s}}$ & 0.006 & -0.019 & 0.7059 & -1.3103 & 0.9911 \\
\hline & $\frac{\bar{\mu}}{\mu_{s}}$ & 0.0139 & 0.3759 & 0.958 & -1.577 & 1.2248 \\
\hline
\end{tabular}

In addition to the results above, we develop here close-form expressions that predict the magnetic permeability of planar cellular solids. These relations are similar to the formulas given for the effective thermal conductivity and dielectric coefficient of porous media [60, 61]: 
- Parallel: $\mu=\rho_{r} \mu_{s}+\left(1-\rho_{r}\right) \mu_{g}$

- Series: $\mu=\frac{\mu_{s} \mu_{g}}{\mu_{g} \rho_{r}+\mu_{s}\left(1-\rho_{r}\right)}$

- Geometric mean: $\mu=\mu_{s}^{\rho_{r}} \mu_{g}^{\left(1-\rho_{r}\right)}$

- Hashin-Shtrikman bounds: $\mu=\mu_{s}+\frac{1-\rho_{r}}{\frac{1}{\mu_{g}-\mu_{s}}+\frac{\rho_{r}}{3 \mu_{s}}}$

$$
\mu=\mu_{g}+\frac{\rho_{r}}{\frac{1}{\mu_{s}-\mu_{g}}+\frac{1-\rho_{r}}{3 \mu_{g}}}
$$

- Iso-magnetic bounds for a planar square cell (analogous to Crane-Vachon model [62]), with a unit length, strut thickness $t$, and relative density $\rho_{r}=1-(1-t)^{2}$ :

$$
\begin{aligned}
& \mu=\left(\frac{t}{\mu_{s}}+\frac{1-t}{t \mu_{s}+t(t-1) \mu_{g}}\right)^{-1} \quad \text { (Iso-magnetic potential) (5.5a) } \\
& \mu=\mu_{s} t+\frac{\mu_{g} \mu_{s}(1-t)}{\mu_{g} t+\mu_{s}(1-t)} \quad \text { (Iso-magnetic induction) (5.5a) }
\end{aligned}
$$

where $\mu_{g}=4 \pi \times 10^{-7} \frac{H}{m}$ is the magnetic permeability of free space.

Figures 1 and 2 illustrate the effective magnetoelastic properties of the unit cells here examined under a plane stress condition. Figure 1 presents the effective properties (Young's modulus, Poisson's ratio, and shear modulus) obtained numerically via standard mechanics. Figure 2 compares the effective magnetic permeability obtained via standard mechanics homogenization and alternative techniques, i.e. the micromechanical models presented in Eqs. (5.1) - (5.5). The results show the role of cell topology on the magnetic permeability for electrically conducting cellular solids. Figure $2 \mathrm{a}$ compares the normalized magnetic permeability predicted by the following micromechanics models: parallel, series, and geometric mean models, Hashin bounds, iso-magnetic potential/induction bounds, and standard mechanics homogenization. Compared to the results from standard mechanics homogenization, Fig. 2a shows that the parallel micromechanics model overpredicts the magnetic permeability as opposed to the series model, which underpredicts them. Because of its simplicity, the parallel model has been used widely in the literature for predicting magnetic properties. Our numerical results, however, shows that a discrepancy of up to $75.5 \%$ can be observed between the effective magnetic permeability predicted by the parallel model considered in reference [37] and the standard mechanics homogenization, which is our benchmark model here. In addition, Fig. 2a demonstrates that the iso-magnetic potential/induction model provides closer bounds to the results obtained from the standard mechanics homogenization, than those provided by the Hashin bounds. 


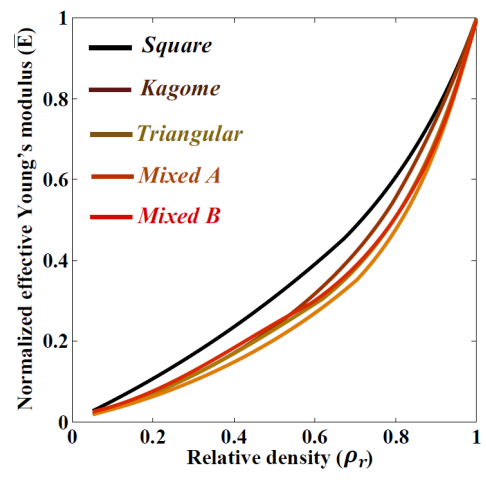

(a)

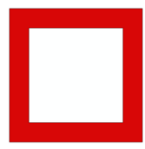

Square

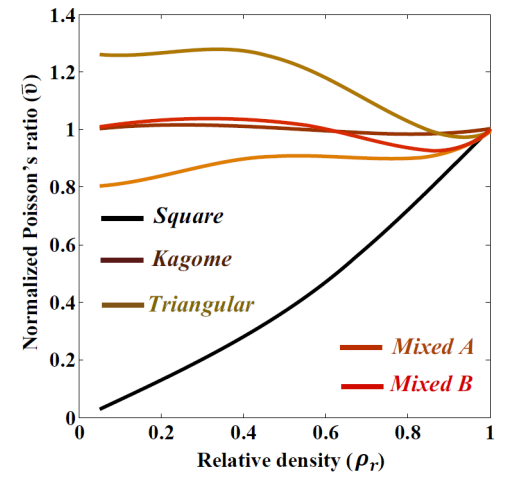

(b)

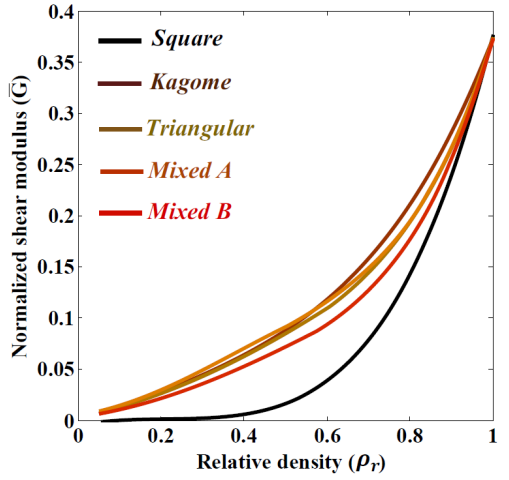

(c)
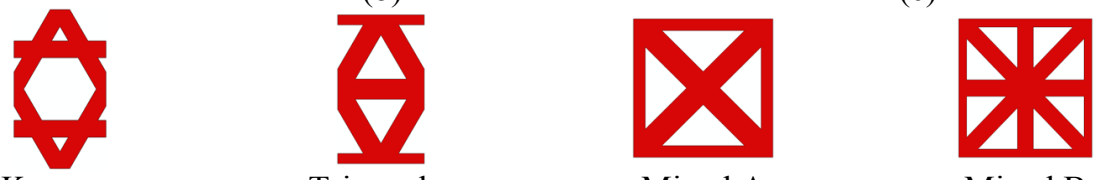

Triangular

Mixed A

Mixed B

Fig. 1. Normalized effective (a) Young's modulus, (b) Poisson's ratio, and (c) shear modulus for plane stress lattices [38].

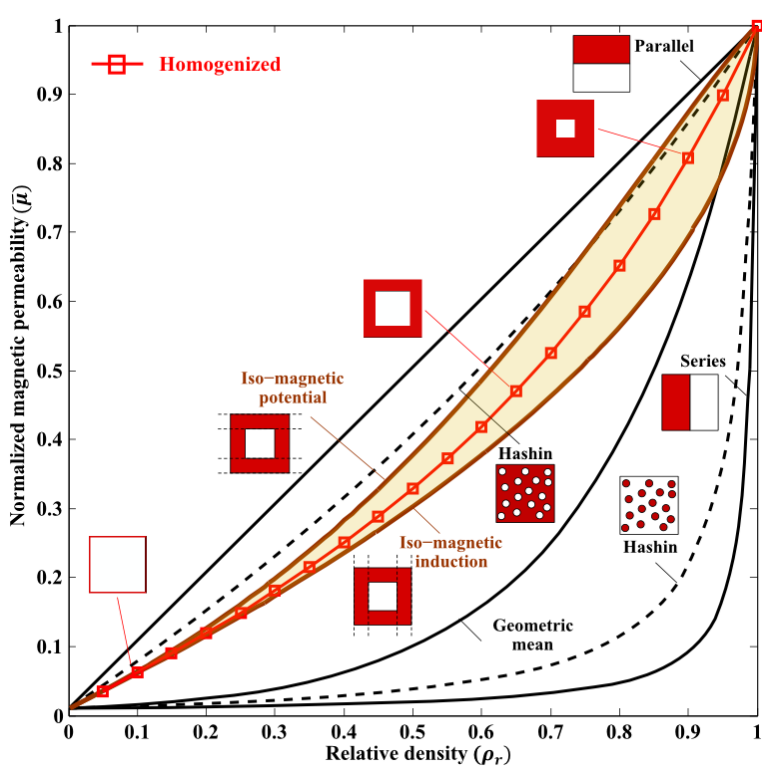

(a)

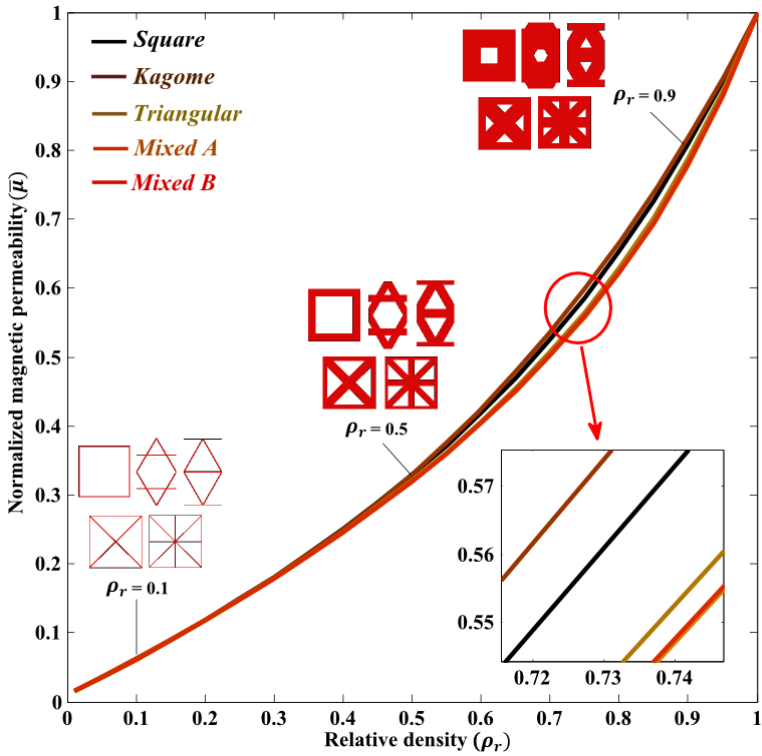

(b)

Fig. 2. Normalized magnetic permeability for the whole range of relative density: (a) effect of micromechanics models and (b) effect of cell topology (Cell topology transition occurs at $\rho_{r}=0.65$ for Kagome lattices).

As seen in Fig. 2b, the effective magnetic permeability of periodic cellular solids is mainly governed by the relative density $\rho_{r}$; however, the cell topology can also play a role. Kagome and mixed A cell topologies draw, respectively, the upper and lower bounds of the magnetic permeability among the planar lattices under investigation. The maximum discrepancy of 
$7.8 \%$ is found between the magnetic permeability of the Kagome and mixed A cell topologies. The following sections examine specifically the role of relative density and cell topology on the dynamic responses of an electrically conducting sandwich cylinder.

\section{Problem Definition}

We consider a multilayered infinitely long cylinder for which we can assume plane strain, and a multilayered thin disk which satisfies the condition of plane stress. The cylinder (or disk) is electrically conducting and subjected to a constant external magnetic field, $H_{z}$, parallel to the out of plane axis (Fig. 3). A dynamic eigenstrain is assumed within an arbitrary layer. The outer radius of the $k$-th layer is represented by $R_{k}(k=1,2, \cdots, N)$, where $N$ is the total number of layers.

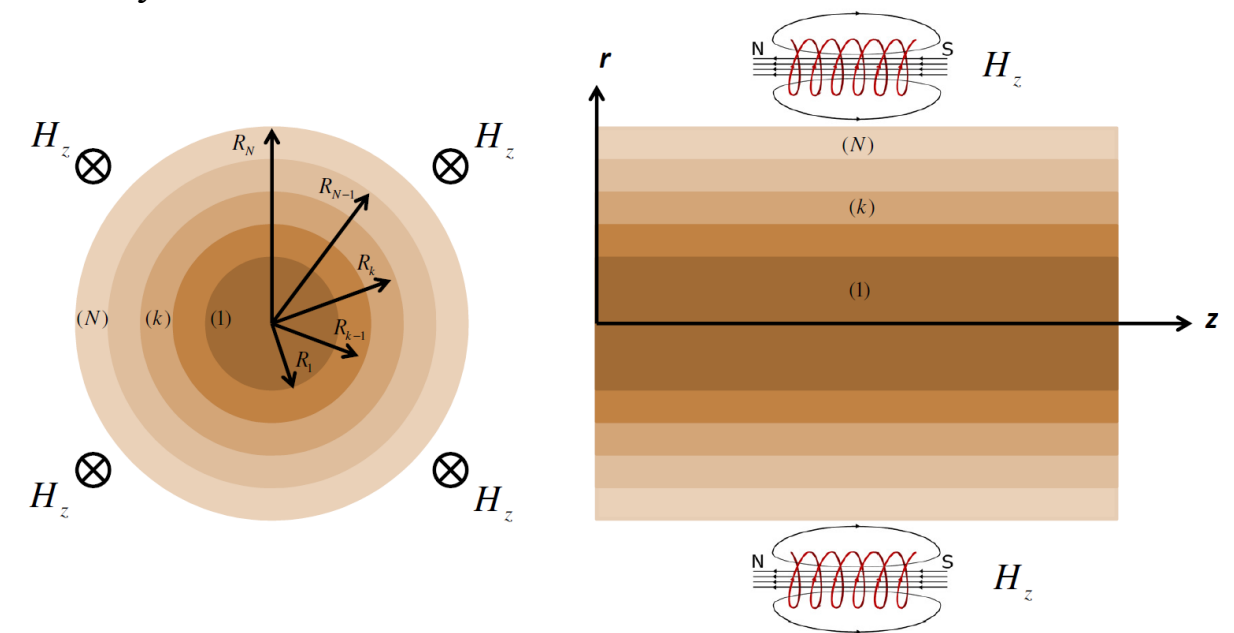

Fig. 3 A multilayered infinitely long cylinder or thin disk under a prescribed magnetic field.

It is assumed that the $k$-th layer undergoes a dynamic eigenstrain, $\varepsilon_{m n}^{*(k)}$, with a predetermined distribution in the radial direction [2]:

$$
\varepsilon_{m n}^{*(k)}(r, t)=\delta_{m n} E^{*(k)}(r) \phi(t)
$$

where the superscript $k$ corresponds to the $k$-th layer, $\delta_{m n}(m, n=1,2,3)$ is the Kronecker delta function, $r$ represents the radial coordinate, and $t$ is time. In this paper, the predetermined spatial distribution of the eigenstrain along the radial direction is assumed by an arbitrary $L^{t h}$-order polynomial as $E^{*(k)}(r)=\sum_{l=0}^{L} A_{l}^{(k)} r^{l}$ with arbitrary coefficients $A_{l}^{(k)}$ $(l=0,1, \ldots, L) ; \phi(t)$ is the temporal function of the time-dependent eigenstrain. We recall that $\varepsilon_{m n}^{*(k)}$ might assume a special-order inelastic strain distribution, e.g. hygrothermal, if the 
material is heterogeneous and/or anisotropic [15]. The relation of the elastic strain, $\varepsilon_{m n}^{(k)}$, eigenstrain, $\varepsilon_{m n}^{*(k)}$, and the total strain, $e_{m n}^{(k)}$, is [2]:

$$
e_{m n}^{(k)}=\varepsilon_{m n}^{(k)}+\varepsilon_{m n}^{*(k)}
$$

The constitutive equation for an isotropic material is written as [2]:

$$
\sigma_{m n}^{(k)}=2 G^{(k)}\left(\varepsilon_{m n}^{(k)}+\frac{v^{(k)}}{1-2 \nu^{(k)}} \varepsilon^{(k)} \delta_{m n}\right)
$$

where $\sigma_{m n}^{(k)}$ and $v^{(k)}$ are stress components and Poisson's ratio, respectively; $\varepsilon^{(k)}=\varepsilon_{m m}^{(k)}$ represents the volumetric strain expressed with the Einstein's tensorial convention. Furthermore, $G^{\prime(k)}=\frac{E^{(k)}}{2\left(1+v^{(k)}\right)}$, where $E^{(k)}$ is the Young's modulus.

For an axisymmetric electrically conducting cylinder/disk under a constant external magnetic field $H_{z}$ [63], the equation of motion is:

$$
\sigma_{r r, r}^{(k)}+\frac{\sigma_{r r}^{(k)}-\sigma_{\theta \theta}^{(k)}}{r}+\mu^{(k)} H_{z}^{2}\left(u_{, r}^{(k)}+\frac{u^{(k)}}{r}\right)_{, r}=\rho^{(k)} \ddot{u}^{(k)}
$$

where $\rho^{(k)}$ is density, $\mu^{(k)}$ is the magnetic permeability, and $u^{(k)}$ is the radial displacement. The symbol "double over-dots" represents the second-order derivative with respect to time and a subscript comma denotes the differentiation with respect to the radial coordinate. From Eqs. (6) to (9), we can obtain the differential equation for a radially distributed eigenstrain of an arbitrary order of polynomial:

where

$$
u_{, r r}^{(k)}+\frac{u_{, r}^{(k)}}{r}-\frac{u^{(k)}}{r^{2}}+a^{(k)} \ddot{u}^{(k)}=-b^{(k)}\left(\sum_{l=1}^{L} l A_{l}^{(k)} r^{l-1}\right) \varphi(t)
$$

$$
\begin{aligned}
& a^{(k)}= \begin{cases}\frac{\rho^{(k)}\left(2 v^{(k)}-1\right)}{2 G^{(k)}\left(1-v^{(k)}\right)+\mu^{(k)} H_{z}^{2}\left(1-2 v^{(k)}\right)}, & \text { plane strain } \\
\frac{\rho^{(k)}\left(v^{(k)}-1\right)}{2 G^{\prime(k)}+\mu^{(k)} H_{z}^{2}\left(1-v^{(k)}\right)}, & \text { plane stress }\end{cases} \\
& b^{(k)}= \begin{cases}2 G^{\prime(k)}\left(1+v^{(k)}\right) & \text { plane strain } \\
-\frac{2 G^{\prime(k)}\left(1-v^{(k)}\right)+\mu^{(k)} H_{z}^{2}\left(1-2 v^{(k)}\right)}{2(k)}\left(1+v^{(k)}\right) & \text { plane stress } \\
-\frac{2 G^{\prime(k)}+\mu^{(k)} H_{z}^{2}\left(1-v^{(k)}\right)}{2 G^{(k)}} & \end{cases}
\end{aligned}
$$

\section{Methodology}


The multilayered cylinder, presented in Section 3, could contain layers of solid or cellular materials. The effective magnetoelastic properties, obtained in Section 2 are used here for the layers made of a cellular material. As an example, Fig. 4 depicts a simplified homogenization scheme for a cellular layer, sandwiched between two solid layers.

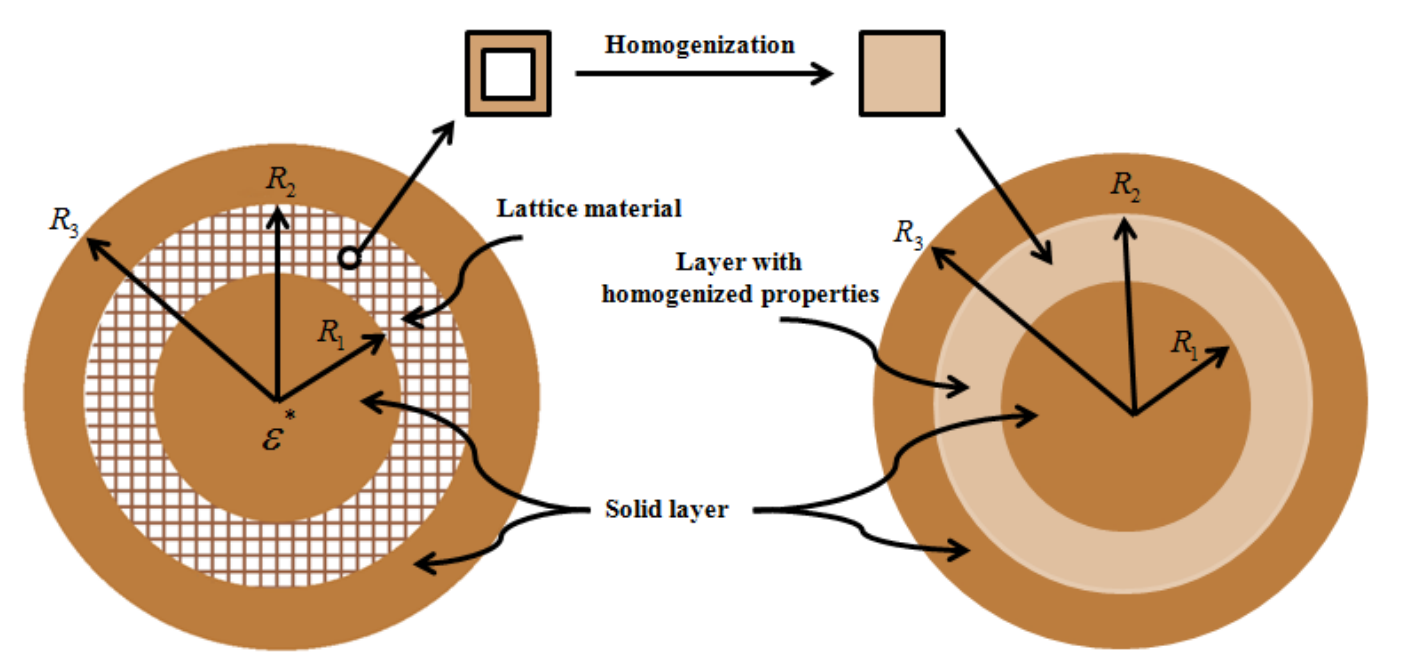

Fig. 4. Sandwich cylinder with a middle layer of square lattice (left) and its homogenized properties (right).

Herein, we present a methodology for the dynamic analysis of sandwich cylinders/disks undergoing a time-harmonic or a transient eigenstrain. For time-harmonic analysis, the time function is assumed as $\varphi(t)=e^{-j \omega t}$, where the complex variable is $j=\sqrt{-1}$ and $\omega$ stands for the angular frequency of the harmonic eigenstrain.

Being $\varphi(t)=e^{-j \omega t}$, the resultant elastic fields written for a time-harmonic eigenstrain at frequency $\omega$ are:

$$
\begin{gathered}
u^{(k)}(r, t)=\bar{u}^{(k)}(r) e^{-j \omega t} \\
\sigma_{r r}^{(k)}(r, t)=\bar{\sigma}_{r r}^{(k)}(r) e^{-j \omega t}, \sigma_{\theta \theta}^{(k)}(r, t)=\bar{\sigma}_{\theta \theta}^{(k)}(r) e^{-j \omega t}, \sigma_{z z}^{(k)}(r, t)=\bar{\sigma}_{z z}^{(k)}(r) e^{-j \omega t}
\end{gathered}
$$

By substituting Eq. (12a) into Eq. (10), the governing equation is reduced to:

$$
u_{, r r}^{(k)}+\frac{u_{, r}^{(k)}}{r}-\frac{\left(1+a^{(k)} \omega^{2} r^{2}\right) u^{(k)}}{r^{2}}=-b^{(k)}\left(\sum_{l=1}^{L} l A_{l}^{(k)} r^{l-1}\right)
$$

The solution of Eq. (13) can be obtained as:

$$
\begin{aligned}
& \bar{u}^{(k)}=C_{1}^{(k)} J_{1}\left(\sqrt{-a^{(k)}} \omega r\right)+C_{2}^{(k)} Y_{1}\left(\sqrt{-a^{(k)}} \omega r\right)+\frac{\pi b^{(k)} A_{1}^{(k)}}{2 a^{(k)} \omega^{2}} H_{1}\left(\sqrt{-a^{(k)}} \omega r\right) \\
& -b^{(k)} \sum_{l=2 i+2}^{L} \frac{l A_{l}^{(k)} S_{l, 1}\left(\sqrt{-a^{(k)}} \omega r\right)}{\left(-a^{(k)} \omega^{2}\right)^{(l+1) / 2}}-b^{(k)} \sum_{l=2 i+3}^{L} \frac{l A_{l}^{(k)} s_{l, 1}\left(\sqrt{-a^{(k)}} \omega r\right)}{\left(-a^{(k)} \omega^{2}\right)^{(l+1) / 2}}, \quad i=0,1,2, \cdots
\end{aligned}
$$


where $C_{1}^{(k)}$ and $C_{2}^{(k)}$ are unknown coefficients, determined from the boundary conditions; $J$ and $Y$ are the Bessel functions of the first and second kind, respectively. The Struve function $H_{\alpha}$ and the Lommel functions $S_{\mu, v}$ and $s_{\mu, v}$, in Eq. (14), are defined as [64]:

$$
\begin{gathered}
H_{\alpha}(x)=\sum_{i=0}^{\infty} \frac{(-1)^{i}\left(\frac{x}{2}\right)^{2 i+\alpha+1}}{\Gamma\left(\frac{1}{2}(2 i+3)\right) \Gamma\left(\frac{1}{2}(2 i+2 \alpha+3)\right)} \\
s_{\mu, \nu}(x)=x^{\mu-1} \sum_{i=0}^{\infty} \frac{(-1)^{i} \Gamma\left(\frac{1}{2}(\mu-v+1)\right) \Gamma\left(\frac{1}{2}(\mu+v+1)\right)\left(\frac{x}{2}\right)^{2 i+2}}{\Gamma\left(\frac{1}{2}(\mu-v+2 i+3)\right) \Gamma\left(\frac{1}{2}(\mu+v+2 i+3)\right)} \\
S_{\mu, \nu}(x)=s_{\mu, \nu}(x)+2^{\mu-1} \Gamma\left(\frac{1}{2}(\mu-v+1)\right) \Gamma\left(\frac{1}{2}(\mu+v+1)\right) \\
\times\left\{\sin \left[\frac{1}{2}(\mu-v) \pi\right] J_{v}(x)-\cos \left[\frac{1}{2}(\mu-v) \pi\right] Y_{v}(x)\right\}
\end{gathered}
$$

It is worth mentioning that the solutions obtained in references [21] and [37] are only applicable to eigenstrain with a polynomial distribution of the third-order polynomial. The current closed-form solutions provided in Eq. (14), on the other hand, could be used for a radially distributed eigenstrain with a polynomial of an arbitrary order. As a result, Eq. (14) could be employed along with a Taylor series expansion to obtain closed-form solutions for eigenstrain problems with any form of eigenstrain distribution.

Using Eq. (14), the radial stress for plane strain condition can be obtained as:

$$
\begin{aligned}
& \bar{\sigma}_{r r}^{(k)}(r)=\frac{2 G^{(k)} C_{1}^{(k)}}{1-2 v^{(k)}}\left[f_{1}^{(k)} f_{2}^{(k)} J_{0}\left(f_{1}^{(k)} r\right)+\frac{f_{3}^{(k)}}{r} J_{1}\left(f_{1}^{(k)} r\right)\right]+\frac{2 G^{\prime(k)} C_{2}^{(k)}}{1-2 v^{(k)}}\left[f_{1}^{(k)} f_{2}^{(k)} Y_{0}\left(f_{1}^{(k)} r\right)+\frac{f_{3}^{(k)}}{r} Y_{1}\left(f_{1}^{(k)} r\right)\right] \\
& -\frac{2 G^{(k)} b^{(k)}}{1-2 v^{(k)}} \sum_{l=2 i+2}^{L} \frac{l A_{l}^{(k)}}{\left(f_{1}^{(k)}\right)^{l+1}}\left[l f_{1}^{(k)} f_{2}^{(k)} S_{l-1,0}\left(f_{1}^{(k)} r\right)+\frac{f_{3}^{(k)}}{r} S_{l, 1}\left(f_{1}^{(k)} r\right)\right] \\
& -\frac{2 G^{(k)} b^{(k)}}{1-2 v^{(k)}} \sum_{l=2 i+3}^{L} \frac{l A_{l}^{(k)}}{\left(f_{1}^{(k)}\right)^{l+1}}\left[l f_{1}^{(k)} f_{2}^{(k)} S_{l-1,0}\left(f_{1}^{(k)} r\right)+\frac{f_{3}^{(k)}}{r} s_{l, 1}\left(f_{1}^{(k)} r\right)\right] \\
& -\frac{\pi G^{(k)} b^{(k)} A_{1}^{(k)}}{\left(1-2 v^{(k)}\right)\left(f_{1}^{(k)}\right)^{2}}\left[f_{1}^{(k)} f_{2}^{(k)} H_{0}\left(f_{1}^{(k)} r\right)+\frac{f_{3}^{(k)}}{r} H_{1}\left(f_{1}^{(k)} r\right)\right]-\frac{2 G^{(k)}\left(v^{(k)}+1\right)}{1-2 v^{(k)}} \sum_{l=0}^{L} A_{l}^{(k)} r^{l}
\end{aligned}
$$

where $f_{1}^{(k)}=\sqrt{-a^{(k)}} \omega, f_{2}^{(k)}=1-v^{(k)}$, and $f_{3}^{(k)}=2 v^{(k)}-1$. For a plane stress condition, the radial stress is: 


$$
\begin{aligned}
& \bar{\sigma}_{r r}^{(k)}(r)=\frac{2 G^{(k)} C_{1}^{(k)}}{1-v^{(k)}}\left[f_{1}^{(k)} J_{0}\left(f_{1}^{(k)} r\right)-\frac{f_{2}^{(k)}}{r} J_{1}\left(f_{1}^{(k)} r\right)\right]+\frac{2 G^{\prime(k)} C_{2}^{(k)}}{1-v^{(k)}}\left[f_{1}^{(k)} Y_{0}\left(f_{1}^{(k)} r\right)-\frac{f_{2}^{(k)}}{r} Y_{1}\left(f_{1}^{(k)} r\right)\right] \\
& -\frac{2 G^{\prime(k)} b^{(k)}}{1-v^{(k)}} \sum_{l=2 i+2}^{L} \frac{l A_{l}^{(k)}}{\left(f_{1}^{(k)}\right)^{l+1}}\left[l f_{1}^{(k)} S_{l-1,0}\left(f_{1}^{(k)} r\right)-\frac{f_{2}^{(k)}}{r} S_{l, 1}\left(f_{1}^{(k)} r\right)\right] \\
& -\frac{2 G^{(k)} b^{(k)}}{1-v^{(k)}} \sum_{l=2 i+3}^{L} \frac{l A_{l}^{(k)}}{\left(f_{1}^{(k)}\right)^{l+1}}\left[l f_{1}^{(k)} S_{l-1,0}\left(f_{1}^{(k)} r\right)-\frac{f_{2}^{(k)}}{r} S_{l, 1}\left(f_{1}^{(k)} r\right)\right] \\
& -\frac{\pi G^{(k)} b^{(k)} A_{1}^{(k)}}{\left(1-v^{(k)}\right)\left(f_{1}^{(k)}\right)^{2}}\left[f_{1}^{(k)} H_{0}\left(f_{1}^{(k)} r\right)-\frac{f_{2}^{(k)}}{r} H_{1}\left(f_{1}^{(k)} r\right)\right]-\frac{2 G^{(k)}\left(v^{(k)}+1\right)}{1-v^{(k)}} \sum_{l=0}^{L} A_{l}^{(k)} r^{l}
\end{aligned}
$$

The hoop and axial stresses could be obtained similarly.

The boundary conditions for a multilayered composite cylinder/disk with imperfectly bonded interfaces can be written as [21]:

$$
\begin{gathered}
\bar{u}^{(1)}(r=0) \text { is finite } \\
\bar{u}^{(d+1)}\left(r=R_{d}\right)-\bar{u}^{(d)}\left(r=R_{d}\right)=\chi^{(d)} \bar{\sigma}_{r r}^{(d)}\left(r=R_{d}\right) \quad(d=1,2, \cdots, N-1) \\
\bar{\sigma}_{r r}^{(d)}\left(r=R_{d}\right)=\bar{\sigma}_{r r}^{(d+1)}\left(r=R_{d}\right) \quad(d=1,2, \cdots, N-1) \\
\bar{\sigma}_{r r}^{(N)}\left(r=R_{N}\right)=0
\end{gathered}
$$

where $\chi^{(d)}$ are the compliance constants describing imperfect interfaces; $\chi^{(d)}=0$ represents perfectly bonded interfaces. It is worth noting that Eq. (18a) leads to $C_{2}^{(1)}=0$ for the first layer due to the singularity of the Bessel function of the second kind at $r=0$.

Substituting Eqs. (14) and (16) or (17) into the interfacial and boundary condition equations (18) results in:

$$
\mathbf{D C}=\mathbf{B}
$$

where $\mathbf{D}$ is a $2 N \times 2 N$ matrix and $\mathbf{B}$ is a $2 N \times 1$ vector, whose components are omitted here for the sake of brevity; $\mathbf{C}$ represents a $2 N \times 1$ vector of unknown coefficients as: $\mathbf{C}=\left[\begin{array}{llllllll}C_{1}^{(1)} & C_{2}^{(1)} & C_{1}^{(2)} & C_{2}^{(2)} & \cdots & C_{2}^{(N-1)} & C_{1}^{(N)} & C_{2}^{(N)}\end{array}\right]^{\mathrm{T}}$, where superscript " $\mathrm{T}$ " represents the transpose of a matrix. From Eq. (19), we determine $C_{1}^{(k)}$ and $C_{2}^{(k)}$, which are used in Eqs. (14), (16), and (17), to obtain the displacement and stress fields. Resonance occurs if the frequency of the harmonic eigenstrain $\omega$ equals the natural frequency, calculated by setting the determinant of the D matrix equal to zero.

For transient analysis, the function of time variation of eigenstrain can be in an arbitrary form $\varphi(t)$. If we assume a Heaviside step function for the time function $(\varphi(t)=H(t))$ and consider the zero initial condition for displacement and velocity, applying the Laplace transform to the governing equation (10) leads to: 


$$
u_{, r r}^{(k)}+\frac{u_{, r}^{(k)}}{r}-\frac{\left(1+a^{(k)} s^{2} r^{2}\right) u^{(k)}}{r^{2}}=-\frac{b^{(k)}}{s}\left(\sum_{l=1}^{L} l A_{l}^{(k)} r^{l-1}\right)
$$

where $s$ represents the Laplace variable. The solution of Eq. (21) can be written as:

$$
\begin{gathered}
\tilde{u}^{(k)}=X_{1}^{(k)} J_{1}\left(\sqrt{a^{(k)}} s r\right)+X_{2}^{(k)} Y_{1}\left(\sqrt{a^{(k)}} s r\right)-\frac{\pi b^{(k)} A_{1}^{(k)}}{2 a^{(k)} s^{3}} H_{1}\left(\sqrt{a^{(k)}} s r\right) \\
-\frac{b^{(k)}}{s} \sum_{l=2 i+2}^{L} \frac{l A_{l}^{(k)} S_{l, 1}\left(\sqrt{a^{(k)}} s r\right)}{\left(\sqrt{a^{(k)}} s\right)^{l+1}}-\frac{b^{(k)}}{s} \sum_{l=2 i+3}^{L} \frac{l A_{l}^{(k)} s_{l, 1}\left(\sqrt{a^{(k)}} s r\right)}{\left(\sqrt{a^{(k)}} s\right)^{l+1}}
\end{gathered}
$$

where $X_{1}^{(k)}$ and $X_{2}^{(k)}$ are unknown coefficients determined by applying the displacement and stress boundary conditions, similar to the time-harmonic analysis. The numerical Laplace inversion via Jacobi polynomial can then be used to retrieve the elastic fields in the time domain $[37,65]$.

\section{Analysis of sandwich cylinders}

In this section, we apply the analysis described above to a three-layer $(N=3)$ sandwich cylinder subjected to a time-harmonic eigenstrain. This scenario might represent, for example, a time-dependent hygrothermal or electromagnetic disturbance. We examine the role of bonding imperfection, relative density, and cell topology on the time-dependent responses. Carbon steel with $E_{s}=200 \times 10^{9} \mathrm{~Pa}, v_{s}=0.29$, and $\rho_{s}=7.87 \times 10^{3} \mathrm{~kg} / \mathrm{m}^{3}$ is chosen as the material for the cylinder, for both the solid skins and the cellular core. The coefficients of the polynomial eigenstrain are assumed unity, $A_{l}^{(k)}(l=0,1,2, \ldots, L)=1$, with identical compliance constants $(\chi)$ describing imperfections at the layer interfaces. The outer radii of the three layers are assumed as $R_{1}=0.25 \mathrm{~m}, R_{2}=0.75 \mathrm{~m}$, and $R_{3}=1 \mathrm{~m}$. Moreover, we introduce the following dimensionless parameters to represent time-harmonic radial displacement, stress components, radial coordinate, the compliance constant of imperfect bonding, and external magnetic field:

$$
U=\frac{u}{R_{N}}, \Sigma_{r r}=\frac{\sigma_{r r}}{E_{s}}, \Sigma_{\theta \theta}=\frac{\sigma_{\theta \theta}}{E_{s}}, \Sigma_{z z}=\frac{\sigma_{z z}}{E_{s}}, \zeta=\frac{r}{R_{N}}, \beta=\frac{E_{s} \chi}{R_{N}}, \alpha=\frac{\mu_{s} H_{z}^{2}}{E_{s}}
$$

\subsection{Role of bonding imperfection}

The effect of bonding imperfection on the static $(\omega=0)$ structural responses of a threelayer, infinitely long cylinder is illustrated in Fig. 5. The eigenstrain applied in its middle layer is described by a quadratic polynomial $\left(E^{*(2)}=r^{2}\right)$, an assumption that allows us to compare and validate the results with those given in a previous study [66]. The cylinder is 
composed of layers of solid material $\left(\rho_{r}=1\right)$ and subjected to a constant magnetic field $\alpha=1$.

As seen in Fig. 5, the radial displacement and radial stress are G0 (positional) continuous for perfectly bonded interfaces $(\beta=0)$; however, the presence of eigenstrain in the middle layer leads the discontinuity of the hoop and axial stresses along the interfaces. These results confirm those reported in the literature [66]. On the other hand, the non-zero compliance constant $\beta$ (bonding defects) leads to discontinuous displacement and G0 continuous radial stress distributions due to the interfacial boundary conditions (Eq. (18)). As shown in Fig. 5, less stiff interfaces (higher $\beta$ ) increase the radial displacement, hoop stress, and axial stress in the middle layer, as opposed to the outer layers. In addition, we observe lower radial stress in the first and second layers of the cylinder, whereas $\beta$ has little influence in the third layer. The stress analysis presented here for imperfectly bonded cylindrical composites under prescribed eigenstrains, such as hygrothermal and residual strains, can capture the stress discontinuity and stress peaks throughout the layers. It can thus be used to verify the occurrence of structural failure in multilayered composites with heterogeneity or residual stress.

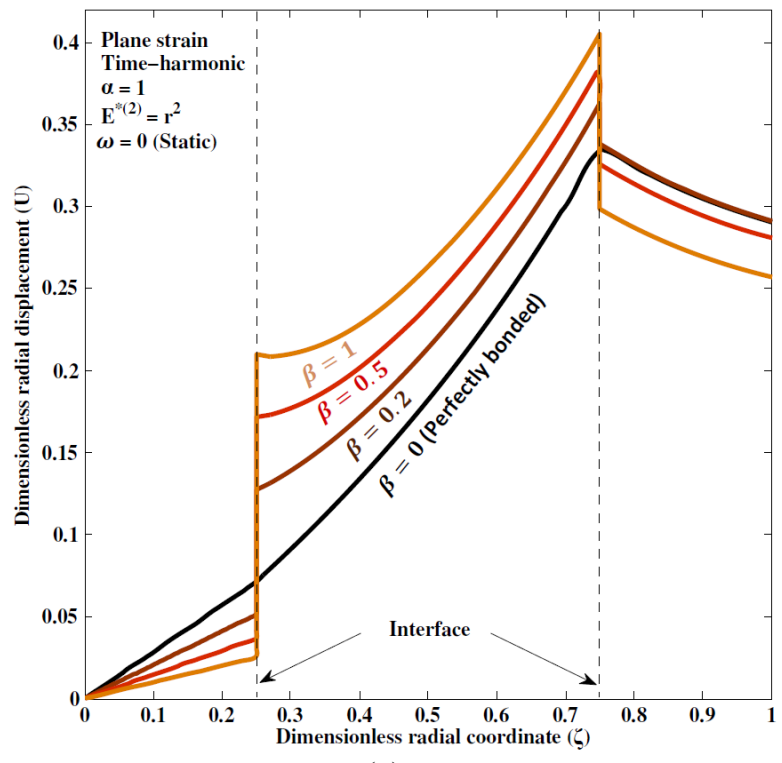

(a)

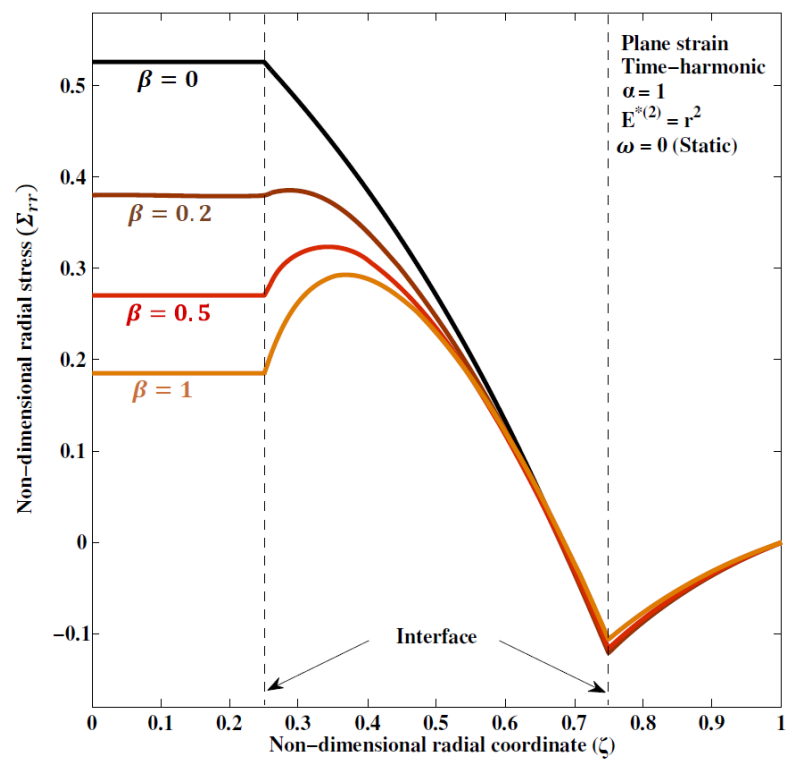

(b) 


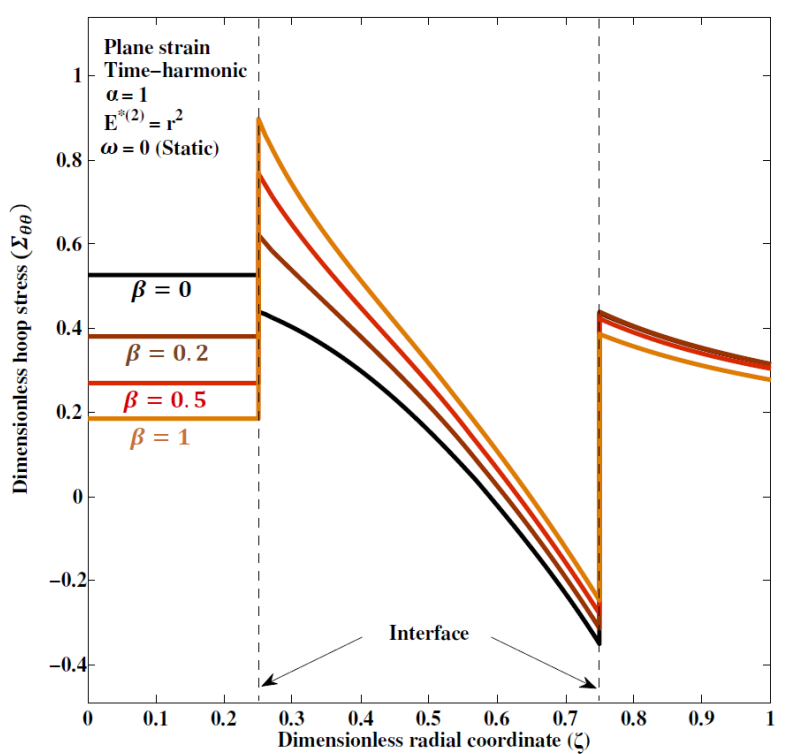

(c)

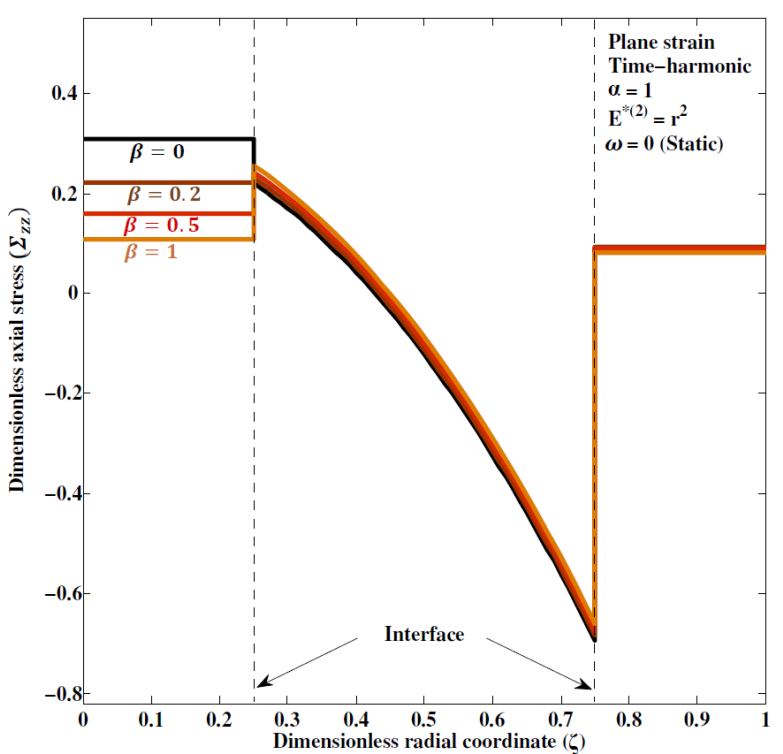

(d)

Fig. 5 Effect of compliance constants, describing imperfect interfaces in a cylinder, on the distribution of: (a) radial displacement, (b) radial stress, (c) hoop stress, and (d) axial stress.

\subsection{Role of relative density}

Assuming a perfect bonding $(\beta=0)$ among the layers, we study here the role of relative density on the time-harmonic responses of a sandwich disk (Fig. 4). The core material is a square lattice, with material properties described in Appendix A. The relative density of the cellular core is $\rho_{r}^{(2)}=\rho_{r}$, while the material of the inner and outer layers (first and third layers) is solid $\left(\rho_{r}^{(1)}=\rho_{r}^{(3)}=1\right)$. The sandwich disk is subjected to a constant magnetic field $\alpha=1$, whereas the inner layer endures a time-dependent eigenstrain. Figure C1, in Appendix $\mathrm{C}$, compares the radial displacement of a sandwich cylinder subjected to time-harmonic eigenstrains with several orders of polynomial distribution. While increasing the order of the polynomial from second to third enhances the maximum value of radial displacement by $1 \%$, the increase is less than $0.2 \%$ if the order of the polynomial rises from third to fourth. This infers that a third-order polynomial is appropriate, and thus is chosen here for the rest of the analysis.

Figures $6 \mathrm{a}$ and $6 \mathrm{~b}$ show the influence of relative density on the distribution of dimensionless, time-harmonic $(\omega=1000 \mathrm{rad} / \mathrm{s})$ radial displacement and von Mises stress $\left(\Sigma_{V M}=\frac{\sigma_{V M}}{E_{s}}\right)$. The domain describing the radial displacement and von Mises stress is shown for a range of relative density $\left(0.01 \leq \rho_{r} \leq 1\right)$. We observe that a reduction in the relative density causes an increase in the maximum radial displacement and a decrease in the von Mises stress within the circular disk. The domain boundaries are set approximately by curves 
for $\rho_{r}=0.01, \rho_{r}=0.7$, and $\rho_{r}=1$ (solid material). These maps offer an insight into the design of sandwich disks, which require satisfying requirements for structural deformation (Fig. 6a) and failure (Fig. 6b).

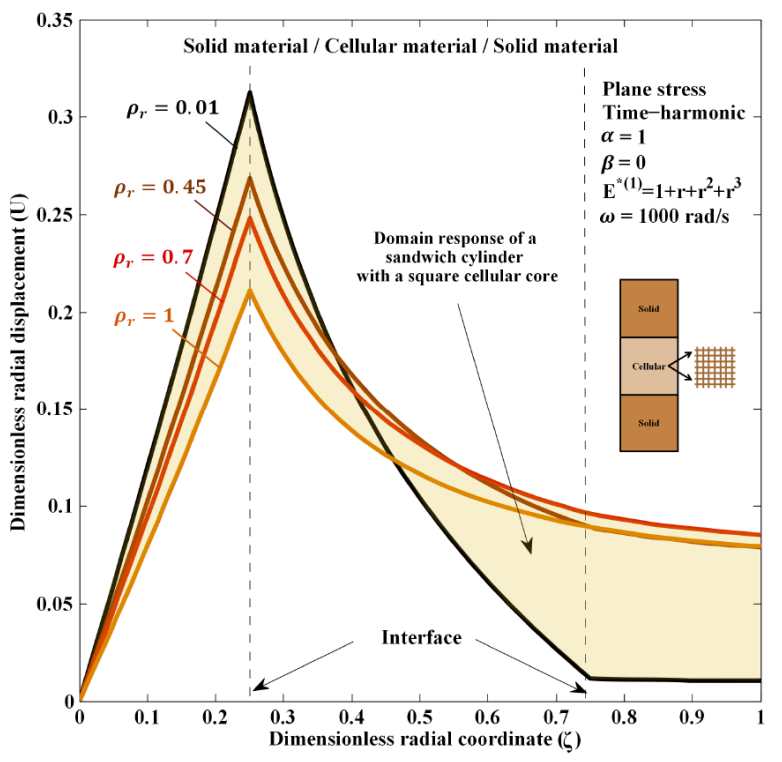

(a)

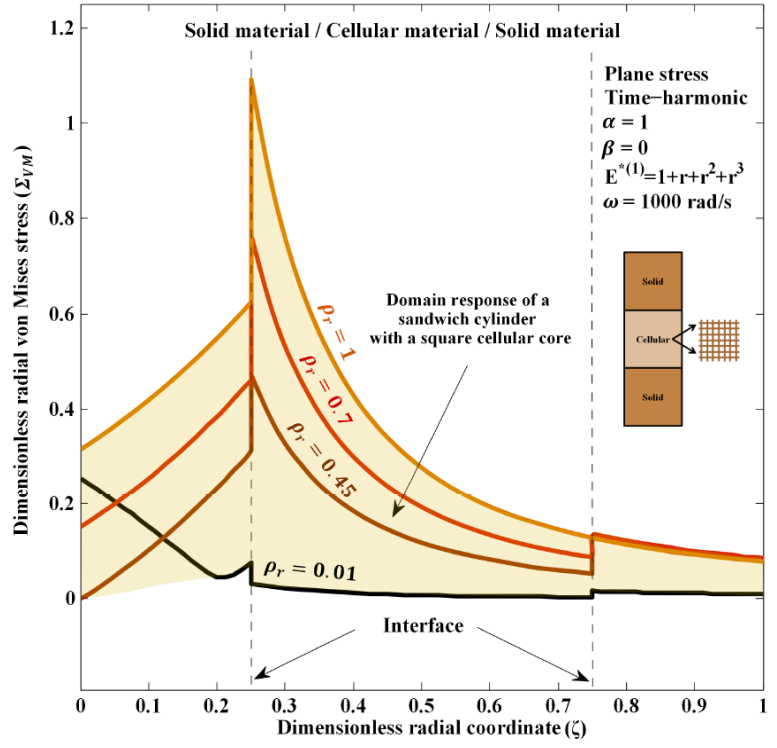

(b)

Fig. 6 Effect of relative density of cellular core on time-harmonic: (a) radial displacement and (b) von Mises stress.

Figure 7 illustrates the role of relative density on the first natural frequency for alternative compliance constants and external magnetic fields. For perfectly bonded sandwich disks, $(\beta=0)$ and in the absence of external magnetic field $(\alpha=0)$, the numerical results are plotted together with those from a finite element analysis via the software package Abaqus Inc. (Dassault Systèmes). As seen in Fig. 7, the theoretical predictions follow the computational results with a maximum error of $1 \%$ at $\rho_{r}=0.6$. Reducing the relative density of the cellular core decreases the weight and first natural frequency, an undesirable outcome for certain applications. Two phases are distinguished in Fig. 7; phase I $(\alpha=0)$ shows the role of the compliance constant of the interfaces and phase II $(\beta=0)$ reveals the effect of the external magnetic field. On one hand, increasing the external magnetic field increases the natural frequency and inhibits the resonance of a sandwich working under a dynamic load with a specific range of frequency. On the other hand, less stiff interfaces undesirably lead to lower natural frequencies, a phenomenon that is negligible for lower values of relative density. 


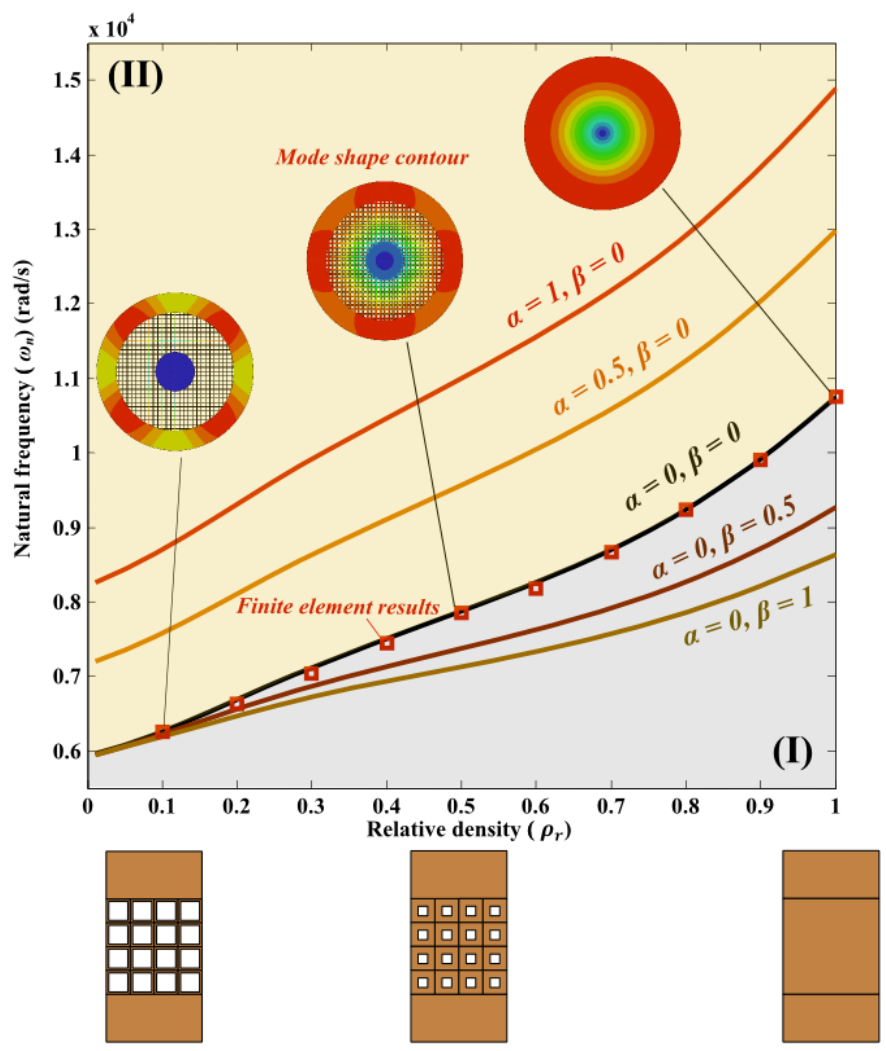

Fig. 7 First natural frequency for a sandwich disk obtained via theory and finite element analysis for the full range of relative density of the square cellular core. Phases I and II show, respectively, the effect of bonding imperfection and external magnetic field.

\subsection{Influence of cell topology}

We examine a sandwich disk with a cellular core of $\rho_{r}=0.2$ and look at the impact of the cell topology of the cellular core on its time-harmonic magnetoelastic responses. A perfect bonding $(\beta=0)$ is assumed among the layers, subjected to a constant magnetic field $\alpha=1$. The inner layer endures a time-harmonic eigenstrain $(\omega=1000 \mathrm{rad} / \mathrm{s})$ with a cubic radial distribution $\left(E^{*(1)}=1+r+r^{2}+r^{3}\right)$. Figures $8 \mathrm{a}$ and $8 \mathrm{~b}$ show the impact of five planar cell topologies on the dimensionless radial displacement and von Mises stress. As shown in Section 2, the cell topology controls the magnetoelastic properties throughout the layers and hence changes the magnetoelastic responses. The choice of a given cell topology has either a beneficial or an adverse impact on the structural responses. On one hand, Fig. 8a shows that a square cell core decreases the maximum displacement by $3.7 \%$, compared to a mixed A cell. On the other hand, the radial displacement of the outer edge of the sandwich disk $\left(U_{R}\right)$ increases by $37.6 \%$ for a square cellular layer. Moreover, compared to the square cell, the mixed A cell decreases by $93.4 \%$ the maximum von Mises stress in the cellular core and increases it by $19.9 \%$ in the solid layers. 


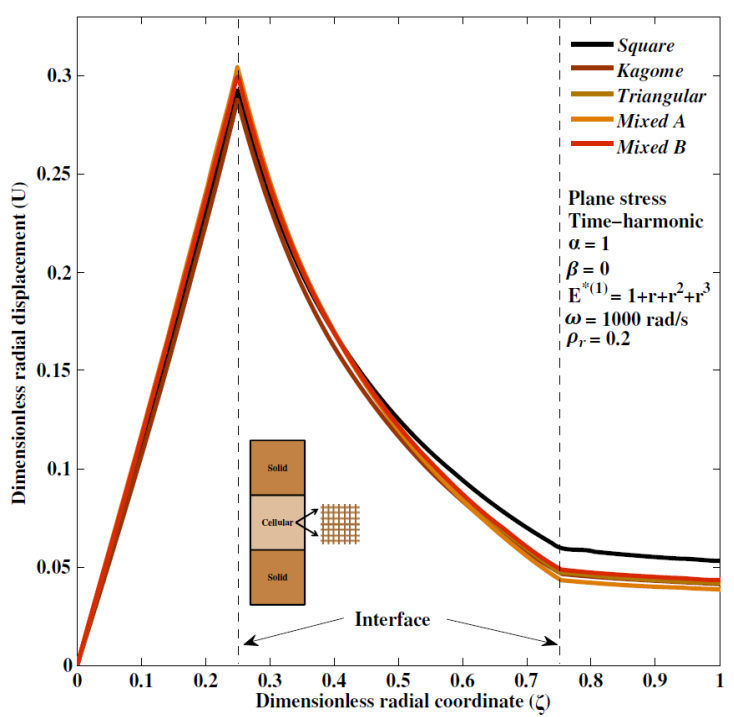

(a)

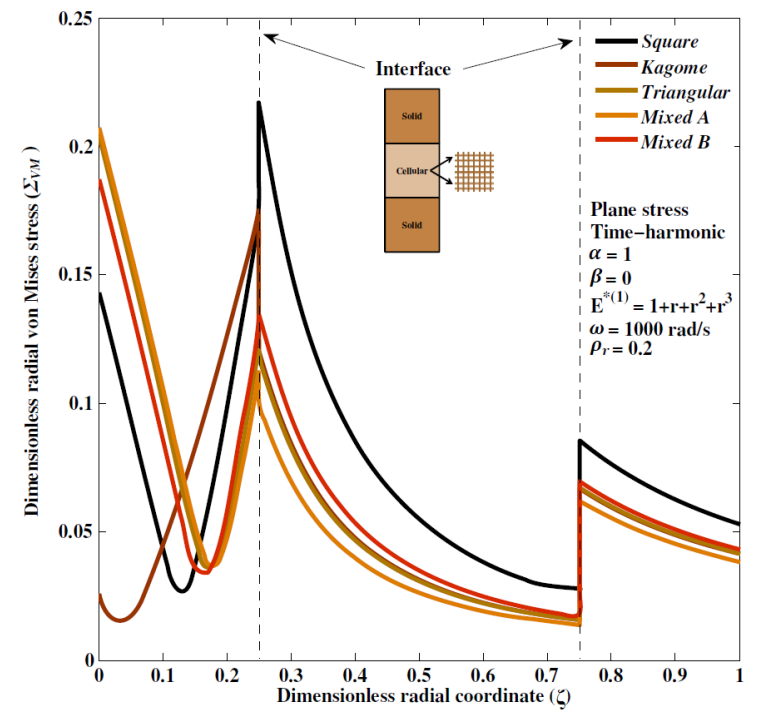

(b)

Fig. 8 Effect of the core cell topology on the time-harmonic: (a) radial displacement, and (b) von Mises stress distributions in a sandwich disk.

Fig. 9a illustrates the role of cell topology on the maximum displacement of an electrically conducting sandwich disk with respect to its outer edge displacement. Similarly, Fig 9b plots the maximum von Mises stress versus the first natural frequency for the whole range of relative density $\left(0.01 \leq \rho_{r} \leq 1\right)$. The displacement and stress contours are also embedded in each figure. As can be seen, all cell topologies follow a similar trend, where an increase in relative density decreases the maximum radial displacement, and increases the radial displacement of the outer edge, the maximum von Mises stress, and the first natural frequency. Cell topology selection for the cellular core can also play a role in the magnitude of each structural response. For instance, the application of a mixed A lattice (point B), rather than a square lattice (point A) as a core of a sandwich disk with $\rho_{r}=0.25$, decreases the displacement of the outer edge and the maximum von Mises stress by $23.3 \%$ and $30.9 \%$, respectively; this comes with an undesirable $2.8 \%$ decrease in the first natural frequency and $4.7 \%$ increase in the maximum radial displacement.

Moreover, Fig. 9 shows the influence of an applied magnetic field on the structural performance of the sandwich disk. For instance, increasing the magnetic field from $\alpha=0$ (point A) to $\alpha=5$ (point $\mathrm{C}$ ) for a sandwich disk with a square lattice of $\rho_{r}=0.25$, favourably increases its natural frequency, with the detriment of making the disk more compliant, and increasing its von Mises stress regime, and the outer edge displacement. The trend does not change for the whole range of relative density of any of the five cell topologies considered in this work. These numerical results show the role played by the applied magnetic field on the structural properties of an electrically conducting sandwich structure. 


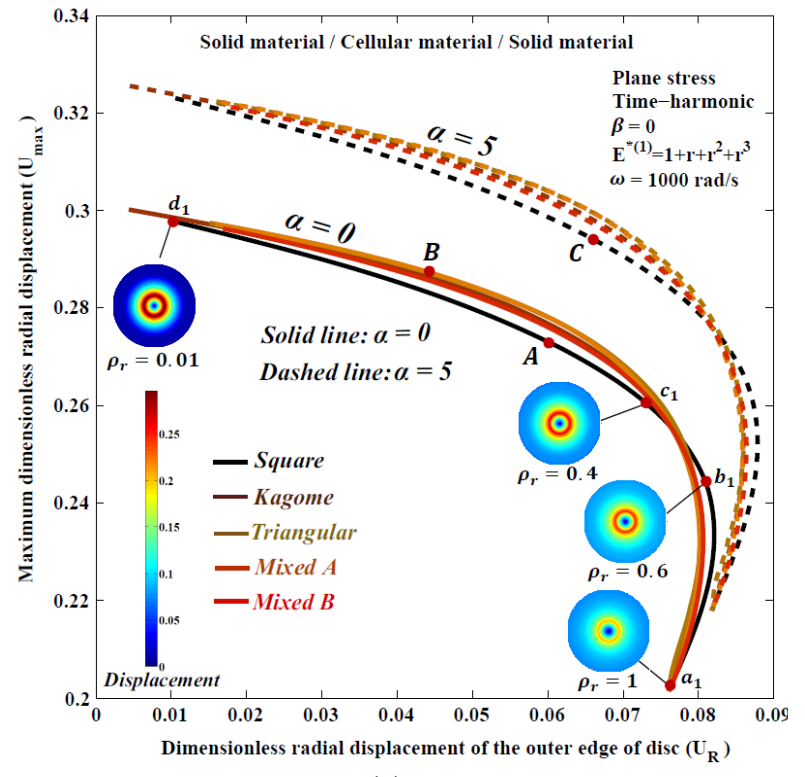

(a)

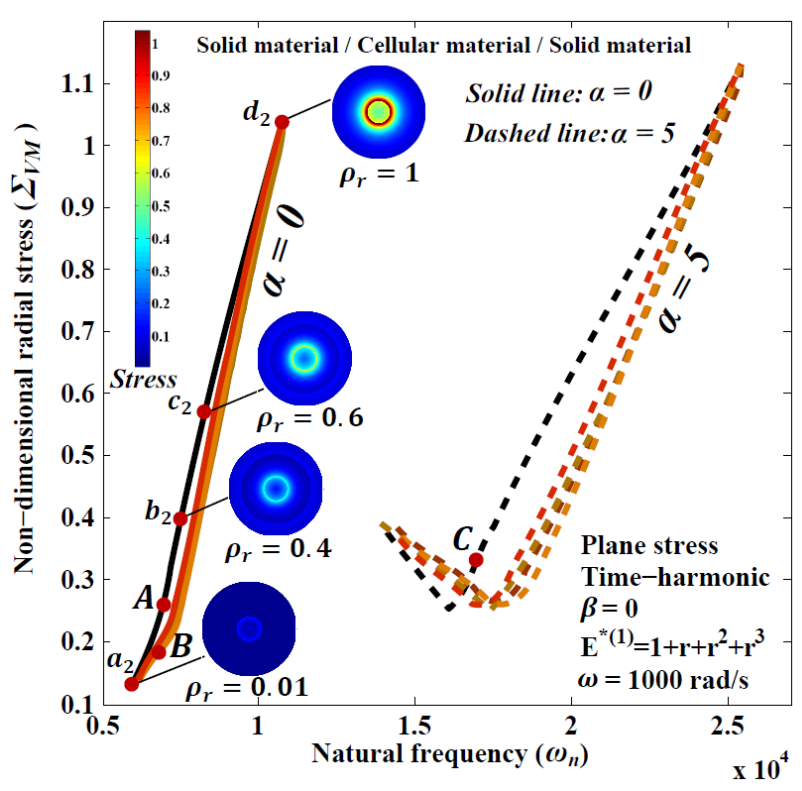

(b)

Fig. 9 Cell topology and relative density of the cellular core govern (a) maximum displacement vs. outer edge displacement and (b) maximum von Mises stress vs. natural frequency; Points A (square lattice with $\alpha=0$ ), B

(mixed A lattice with $\alpha=0$ ), and $\mathrm{C}$ (square lattice with $\alpha=5$ ) refer to sandwich disks with $\rho_{r}=0.25$.

Coordinates of marked points are: $a_{1}\left(U_{R}=0.0761, U_{\max }=0.2014\right), b_{1}\left(U_{R}=0.8098, U_{\max }=0.2442\right)$,

$$
\begin{gathered}
c_{1}\left(U_{R}=0.0733, U_{\max }=0.2603\right), d_{1}\left(U_{R}=0.0099, U_{\max }=0.2978\right), \\
a_{2}\left(\omega_{n}=5960, \sum_{V M}=0.1341\right), b_{2}\left(\omega_{n}=7509, \sum_{V M}=0.3974\right), c_{2}\left(\omega_{n}=8260, \sum_{V M}=0.5678\right), \\
d_{2}\left(\omega_{n}=10760, \sum_{V M}=1.042\right) .
\end{gathered}
$$

Finally the effect of cell topology on the vibratory behavior of a sandwich disk is illustrated in Fig. 10. The harmonic responses in the absence of external magnetic field $(\alpha=0)$ are plotted for the five cell topologies. The perfectly bonded $(\beta=0)$ sandwich disk consists of a cellular core of $\rho_{r}=0.2$. As shown in Fig. 10, the careful selection of the cell topology can postpone the resonance of the sandwich structure effectively and increase the natural frequencies. The influence of the cell topology on the natural frequency is more evident for higher values of the natural frequency. For instance, while the first natural frequency of a sandwich disk with a square lattice core is $3.1 \%$ higher than the mixed A lattice core, for the second natural frequency the increase in natural frequency increases to $21.6 \%$. In addition to cell topology, Appendix D reports the influence of the geometric layout of a sandwich cylinder on its vibratory responses. 


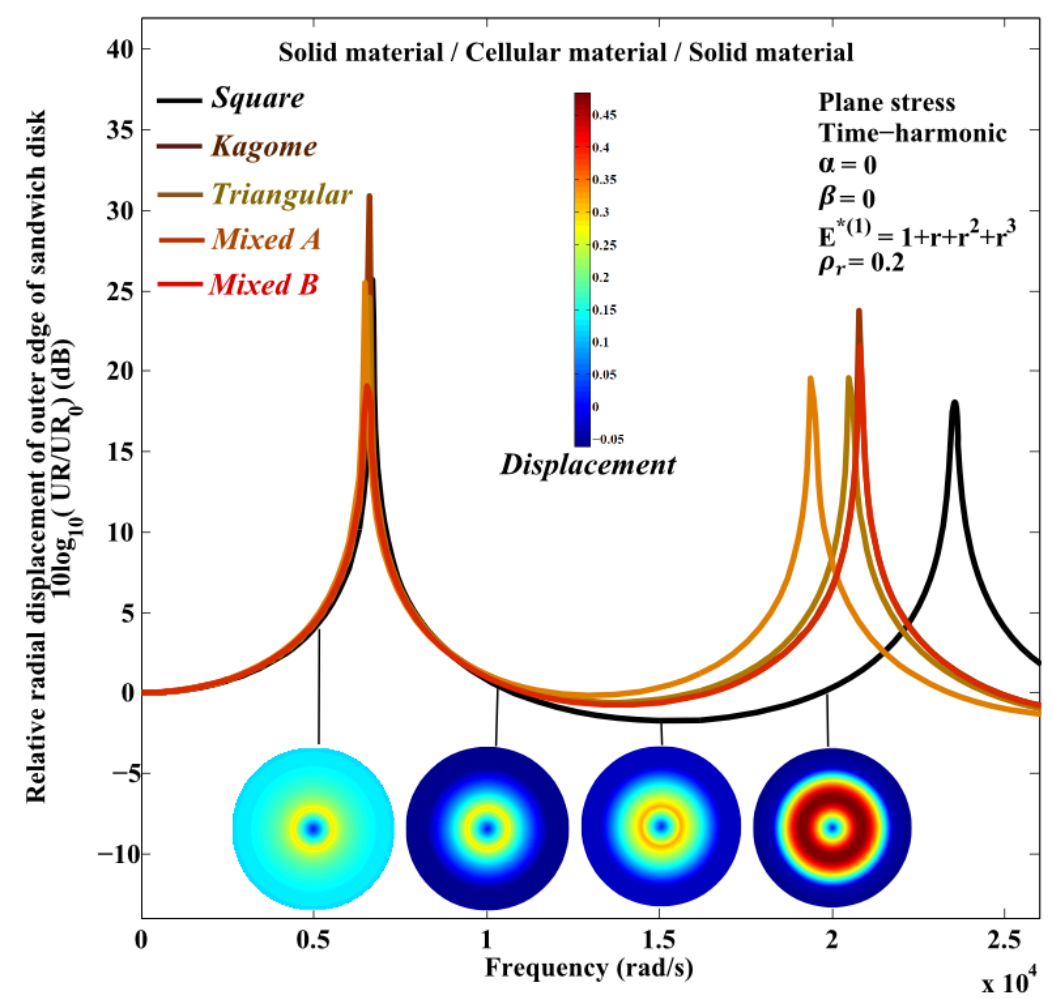

Fig. 10 Effect of cell topology on harmonic responses.

\section{Concluding Remarks}

This paper has examined the magnetoelastic time-dependent response of sandwich cylinders subjected to a dynamic eigenstrain. Time-harmonic and transient eigenstrains have been described by a radial distribution of an arbitrary order polynomial. Closed-form solutions are derived for time-harmonic plane stress/strain conditions, which can be applied for any type of eigenstrain distribution. The effective magnetoelastic properties of the cellular core obtained via standard mechanics homogenization shows the role relative density and cell topology play on displacement, stress distribution, and natural frequency.

The numerical results for sandwich cylinders and disks offer an insight into the role of interfacial bonding, relative density, cell topology, and external magnetic field. The present closed-form solutions can be also useful for the evaluation of the internal and residual stresses caused during the cold-rolled manufacturing and the microfabrication of sandwich structures. From this work, we can draw the following points:

(a) The closed-form solutions here derived can be used to evaluate the harmonic responses of a composite cylinder subjected to any arbitrary distribution of eigenstrain.

(b) The analytic iso-magnetic potential/induction model enables to identify a bound for the effective electromagnetic properties of cellular solids very close to the standard mechanics homogenization results. 
(c) Bonding imperfection drastically increases the maximum hoop stress and the radial displacement within the sandwich cylinder.

(d) An increase of the relative density of the cellular core reduces the maximum radial displacement and raises the radial displacement of the outer edge, the maximum von Mises stress, and the natural frequency.

(e) Careful selection of the cell topology for the core can be used to further improve the structure responses. For example, the application of Mixed A cells instead of squares, decreases the displacement of the outer edge and the maximum von Mises stress by $23.3 \%$ and $30.9 \%$, respectively, while reducing the first natural frequency by $2.8 \%$.

(f) Higher values of the external magnetic field improve the natural frequency of the sandwich and inhibit the resonance without the need to add an extra mass to the system.

\section{Acknowledgments}

A.H. Akbarzadeh acknowledges the financial support by the Natural Sciences and Engineering Research Council of Canada (NSERC).

\section{Appendix A}

Figure A1 illustrates the effect of the eigenstrain distribution on the radial displacement of a sandwich cylinder. The maximum radial displacement occurs at the interface of the inner layer and cellular core. The higher the order of the eigenstrain polynomial, the greater the radial displacement within the sandwich. However, the effect of a higher-order polynomial eigenstrain on the radial displacement decreases with higher order values of the polynomial. For instance, increasing the highest order of a polynomial from second to third increases the maximum value of radial displacement by $1 \%$; the increase is less than $0.2 \%$ when we increase the order of polynomial from third to fourth. This justifies the choice of the thirdorder polynomial used in this paper. 


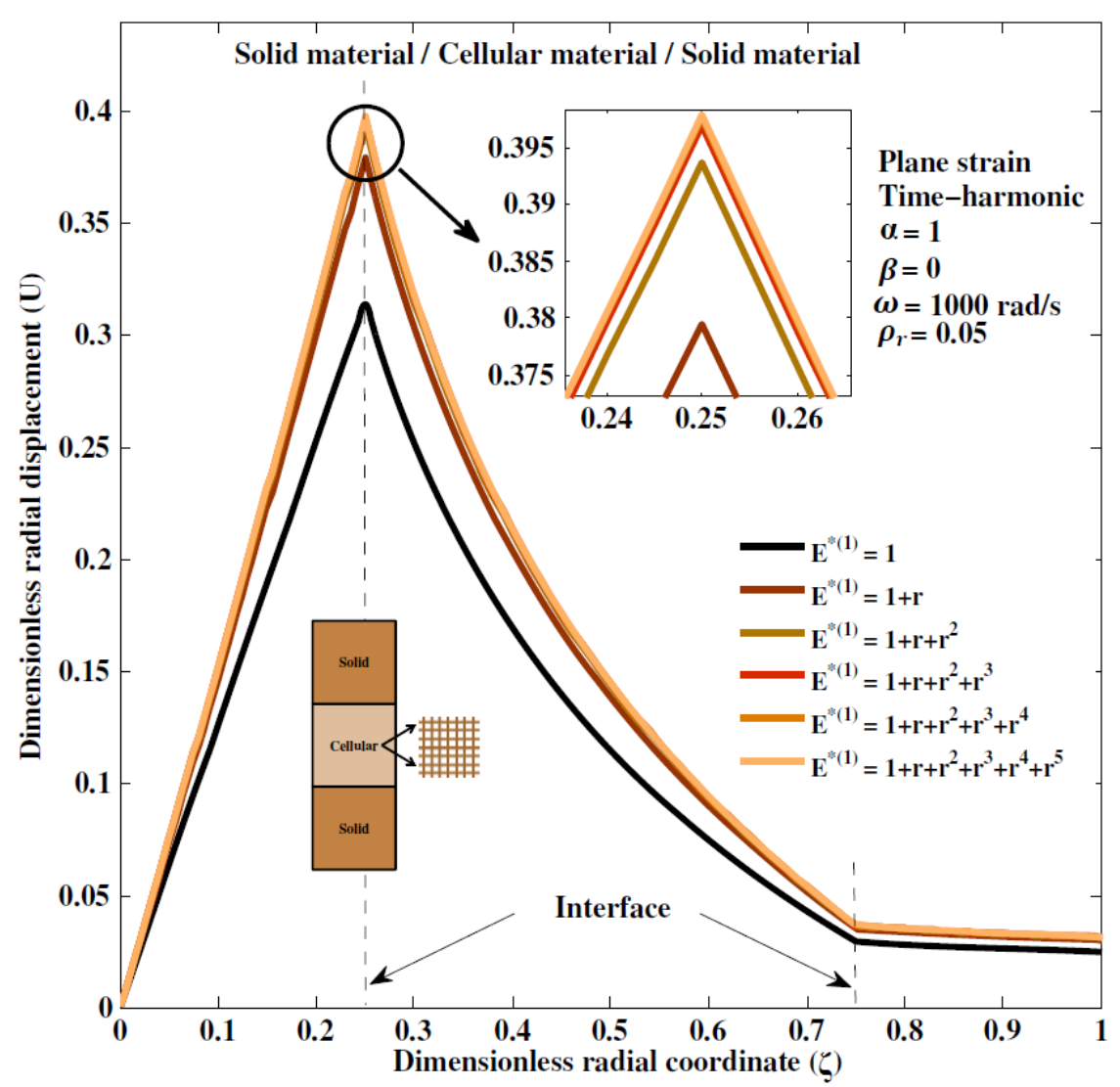

Fig. A1 Radial displacement for alternative radial distributions of eigenstrain.

\section{Appendix B}

Figure B1 shows the role of the geometrical layout of a sandwich disk on the first natural frequency. The disk consists of two layers of solid materials with the thickness of $t_{s}$ $\left(0 \leq t_{s} \leq 0.5\right)$, and a cellular core with the thickness of $t_{c}\left(0 \leq t_{c} \leq 1\right)\left(t_{s}+\frac{t_{c}}{2}=1\right)$. As seen in Fig. B1, increasing $t_{s}$ initially decreases the natural frequency of the sandwich disk; the natural frequency is then increased as $t_{s}$ enhances. Similar trends are observed for alternative cell topologies and relative densities. Among the five cell topologies considered in this paper a sandwich disk with a square lattice core show the highest natural frequency, whereas a core with mixed A cells has the lowest natural frequency. 


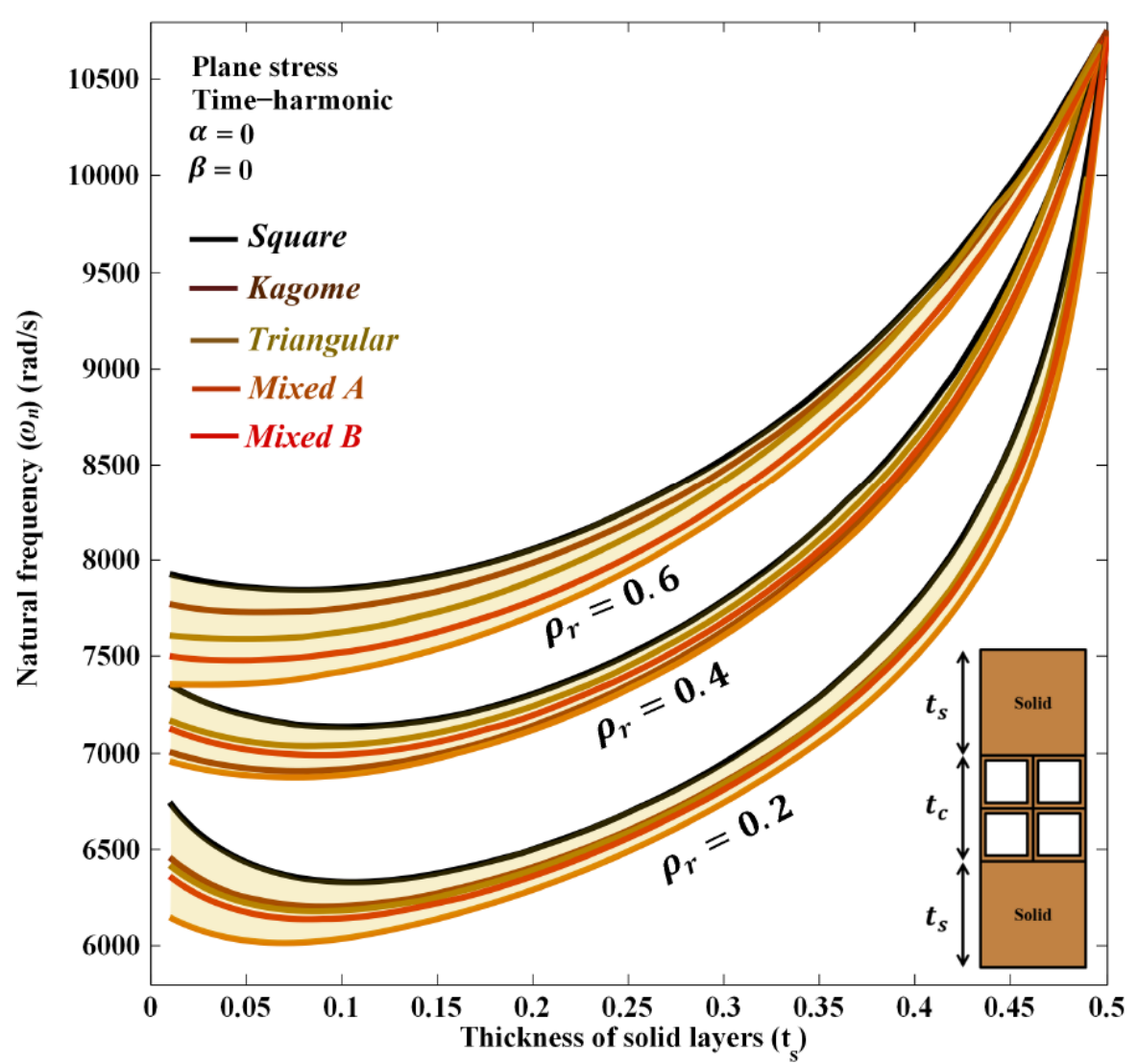

Fig. B1 Role of cell topology of the sandwich core on the first natural frequency plotted against the thickness of the solid layers.

\section{References}

1. Onaka*, S., Elastic strain energy due to deviatoric eigenstrains in an inclusion having symmetric shape. Philosophical magazine letters, 2005. 85(3): p. 115-123.

2. Mura, T., Micromechanics of defects in solids. Vol. 3. 2012: Springer Science \& Business Media.

3. Hensl, T., et al., An eigenstrain approach to predict phase transformation and selfaccommodation in partially stabilized zirconia. Acta Materialia, 2015. 86: p. 361-373.

4. Sun, L., K. Xu, and E. Pan, Inclusion of arbitrary polygon with graded eigenstrain in an anisotropic piezoelectric full plane. International Journal of Solids and Structures, 2012. 49(13): p. 1773-1785.

5. Tirry, W. and D. Schryvers, Linking a completely three-dimensional nanostrain to a structural transformation eigenstrain. Nature materials, 2009. 8(9): p. 752-757.

6. Jun, T.-S. and A.M. Korsunsky, Evaluation of residual stresses and strains using the eigenstrain reconstruction method. International Journal of Solids and Structures, 2010. 47(13): p. 1678-1686. 
7. Matouš, K., Damage evolution in particulate composite materials. International journal of solids and structures, 2003. 40(6): p. 1489-1503.

8. Lokhov, V.A. Stress and Deformation Control by Eigenstrain: Application in Living and Non-Living Systems. in INTERNATIONAL SCIENTIFIC CONFERENCE ON MECHANICS (MECH2012). 2012.

9. Eshelby, J.D. The determination of the elastic field of an ellipsoidal inclusion, and related problems. in Proceedings of the Royal Society of London A: Mathematical, Physical and Engineering Sciences. 1957. The Royal Society.

10. Mura, T. and N. Kinoshita, The polynomial eigenstrain problem for an anisotropic ellipsoidal inclusion. physica status solidi (a), 1978. 48(2): p. 447-450.

11. Li, J.Y., Thermoelastic behavior of composites with functionally graded interphase: a multi-inclusion model. International journal of solids and structures, 2000. 37(39): p. 5579-5597.

12. Adam, C., Eigenstrain induced vibrations of composite plates. Acta mechanica, 2001. 148(1-4): p. 35-53.

13. Lubarda, V., Circular inclusions in anti-plane strain couple stress elasticity. International journal of solids and structures, 2003. 40(15): p. 3827-3851.

14. Nyashin, Y., V. Lokhov, and F. Ziegler, Decomposition method in linear elastic problems with eigenstrain. ZAMM-Journal of Applied Mathematics and Mechanics/Zeitschrift für Angewandte Mathematik und Mechanik, 2005. 85(8): p. 557-570.

15. Sharma, P. and R. Sharma, On the Eshelby's inclusion problem for ellipsoids with nonuniform dilatational Gaussian and exponential eigenstrains. Journal of applied mechanics, 2003. 70(3): p. 418-425.

16. Korsunsky, A.M., G.M. Regino, and D. Nowell, Variational eigenstrain analysis of residual stresses in a welded plate. International Journal of Solids and Structures, 2007. 44(13): p. 4574-4591.

17. Nie, G., et al., Elliptical inhomogeneity in orthotropic composite materials due to uniform eigenstrains. Composites Part B: Engineering, 2008. 39(2): p. 374-385.

18. Jiang, Y., H. Yang, and P. Chen, Analytical study of morphologies for ultra high elastic stiffness of composites with aligned cylindrical fibers. Composite Structures, 2012. 94(8): p. 2390-2396.

19. Yavari, A. and A. Goriely, On the stress singularities generated by anisotropic eigenstrains and the hydrostatic stress due to annular inhomogeneities. Journal of the Mechanics and Physics of Solids, 2015. 76: p. 325-337.

20. Alghamdi, A.A. and A. Dasgupta, Eigenstrain techniques for modeling adaptive structures. I: active stiffness tailoring. Journal of intelligent material systems and structures, 2001. 12(1): p. 31-40.

21. Akbarzadeh, A. and Z. Chen, On the harmonic magnetoelastic behavior of a composite cylinder with an embedded polynomial eigenstrain. Composite Structures, 2013. 106: p. 296-305. 
22. Berbenni, S. and M. Cherkaoui, Homogenization of multicoated inclusion-reinforced linear elastic composites with eigenstrains: Application to thermoelastic behavior. Philosophical Magazine, 2010. 90(22): p. 3003-3026.

23. Liang, X., B. Wang, and Y. Liu, Thickness effect of a thin film on the stress field due to the eigenstrain of an ellipsoidal inclusion. International Journal of Solids and Structures, 2009. 46(2): p. 322-330.

24. Shen, M. and S. Hung, Magnetoelectroelastic analysis of an arbitrary shape inclusion undergoing eigenfields and remote loadings. International Journal of Solids and Structures, 2012. 49(26): p. 3808-3815.

25. Akbarzadeh, A. and D. Pasini, Multiphysics of multilayered and functionally graded cylinders under prescribed hygrothermomagnetoelectromechanical loading. Journal of Applied Mechanics, 2014. 81(4): p. 041018.

26. Wang, X. and Y. Gong, An elastodynamic solution for multilayered cylinders. International journal of engineering science, 1992. 30(1): p. 25-33.

27. Lipton, R., An isoperimetric inequality for the torsional rigidity of imperfectly bonded fiber reinforced cylinders. Journal of elasticity, 1999. 55(1): p. 1-10.

28. Nairn, J., Generalized shear-lag analysis including imperfect interfaces. In other words, 2004. 10: p. 1.

29. Wang, A.-J. and D. McDowell, In-plane stiffness and yield strength of periodic metal honeycombs. Journal of Engineering Materials and Technology, 2004. 126(2): p. 137156.

30. Vigliotti, A. and D. Pasini, Stiffness and strength of tridimensional periodic lattices. Computer Methods in Applied Mechanics and Engineering, 2012. 229: p. 27-43.

31. El Ghezal, M.I., Y. Maalej, and I. Doghri, Micromechanical models for porous and cellular materials in linear elasticity and viscoelasticity. Computational Materials Science, 2013. 70: p. 51-70.

32. Khanoki, S.A. and D. Pasini, Fatigue design of a mechanically biocompatible lattice for a proof-of-concept femoral stem. journal of the mechanical behavior of biomedical materials, 2013. 22: p. 65-83.

33. Larsen, U.D., O. Signund, and S. Bouwsta, Design and fabrication of compliant micromechanisms and structures with negative Poisson's ratio. Microelectromechanical Systems, Journal of, 1997. 6(2): p. 99-106.

34. Bart-Smith, H., J. Hutchinson, and A. Evans, Measurement and analysis of the structural performance of cellular metal sandwich construction. International Journal of Mechanical Sciences, 2001. 43(8): p. 1945-1963.

35. Phani, A.S. and N.A. Fleck, Elastic boundary layers in two-dimensional isotropic lattices. Journal of Applied Mechanics, 2008. 75(2): p. 021020.

36. Challagulla, K., A. Georgiades, and A. Kalamkarov, Asymptotic homogenization modeling of thin composite network structures. Composite structures, 2007. 79(3): p. 432-444.

37. Akbarzadeh, A., et al., Dynamic eigenstrain behavior of magnetoelastic functionally graded cellular cylinders. Composite Structures, 2014. 116: p. 404-413. 
38. Arabnejad, S. and D. Pasini, Mechanical properties of lattice materials via asymptotic homogenization and comparison with alternative homogenization methods. International Journal of Mechanical Sciences, 2013. 77: p. 249-262.

39. Elsayed, M.S. and D. Pasini, Multiscale structural design of columns made of regular octet-truss lattice material. International Journal of Solids and Structures, 2010. 47(14): p. 1764-1774.

40. Ashby, M.F. and D. Cebon, Materials selection in mechanical design. Le Journal de Physique IV, 1993. 3(C7): p. C7-1-C7-9.

41. Kumar, R.S. and D.L. McDowell, Multifunctional design of two-dimensional cellular materials with tailored mesostructure. International Journal of Solids and Structures, 2009. 46(14): p. 2871-2885.

42. Wadley, H.N., Multifunctional periodic cellular metals. Philosophical Transactions of the Royal Society of London A: Mathematical, Physical and Engineering Sciences, 2006. 364(1838): p. 31-68.

43. Evans, A.G., J. Hutchinson, and M. Ashby, Multifunctionality of cellular metal systems. Progress in Materials Science, 1998. 43(3): p. 171-221.

44. Schjødt-Thomsen, J. and R. Pyrz. Influence of statistical cell dispersion on the local strain and overall properties of cellular materials. in MATERIALS PROCESSING AND DESIGN: Modeling, Simulation and Applications-NUMIFORM 2004Proceedings of the 8th International Conference on Numerical Methods in Industrial Forming Processes. 2004. AIP Publishing.

45. Nguyen, N.-T., et al., Characterization of composite material properties using eigenstrain method. Advanced Composite Materials, 2012. 21(4): p. 299-313.

46. Liu, X. and N. Liang, Effective elastic moduli of triangular lattice material with defects. Journal of the Mechanics and Physics of Solids, 2012. 60(10): p. 1722-1739.

47. Kim, S., et al., Fabrication and Characterization of Magnetic Microrobots for Three-Dimensional Cell Culture and Targeted Transportation. Advanced Materials, 2013. 25(41): p. 5863-5868.

48. Singh, J., T. Venkatesh, and K. Challagulla, Electromechanical properties of relaxor ferroelectric foams. Applied Physics Letters, 2013. 103(17): p. 172901.

49. Gorkunov, M., et al., Effective magnetic properties of a composite material with circular conductive elements. The European Physical Journal B-Condensed Matter and Complex Systems, 2002. 28(3): p. 263-269.

50. Singh, P., et al. Additive manufacturing of PZT-5H piezoceramic for ultrasound transducers. in Ultrasonics Symposium (IUS), 2011 IEEE International. 2011. IEEE.

51. Mayer, D., et al., Additive manufacturing of active struts for piezoelectric shunt damping. Journal of Intelligent Material Systems and Structures, 2015: p. 1045389X15575090.

52. Lee, C. and J.A. Tarbutton, Electric poling-assisted additive manufacturing process for PVDF polymer-based piezoelectric device applications. Smart Materials and Structures, 2014. 23(9): p. 095044.

53. Paria, G., Magneto-elasticity and magneto-thermo-elasticity. 1966. 
54. Hollister, S.J. and N. Kikuchi, A comparison of homogenization and standard mechanics analyses for periodic porous composites. Computational Mechanics, 1992. 10(2): p. 73-95.

55. Hassani, B. and E. Hinton, A review of homogenization and topology optimization Ihomogenization theory for media with periodic structure. Computers \& Structures, 1998. 69(6): p. 707-717.

56. Christensen, R., Mechanics of cellular and other low-density materials. International Journal of Solids and Structures, 2000. 37(1): p. 93-104.

57. Wang, X. and W. Stronge, Micropolar theory for two-dimensional stresses in elastic honeycomb. Proceedings of the Royal Society of London. Series A: Mathematical, Physical and Engineering Sciences, 1999. 455(1986): p. 2091-2116.

58. Nguyen, V.D. and L. Noels, Computational homogenization of cellular materials. International Journal of Solids and Structures, 2014. 51(11): p. 2183-2203.

59. Kalamkarov, A., et al., Asymptotic homogenization model for $3 D$ grid-reinforced composite structures with generally orthotropic reinforcements. Composite structures, 2009. 89(2): p. 186-196.

60. Collishaw, P. and J. Evans, An assessment of expressions for the apparent thermal conductivity of cellular materials. Journal of materials science, 1994. 29(9): p. 22612273.

61. Carson, J.K., et al., Thermal conductivity bounds for isotropic, porous materials. International Journal of Heat and Mass Transfer, 2005. 48(11): p. 2150-2158.

62. Bart, G.C.J., Thermal conduction in non homogeneous and phase change media. 1994: TU Delft, Delft University of Technology.

63. Akbarzadeh, A.H., M.H. Babaei, and Z.T. Chen, The thermo-electromagnetoelastic behavior of a rotating functionally graded piezoelectric cylinder. Smart Materials and Structures, 2011. 20(6): p. 065008.

64. Gradshtejn, I.S. and I.M. Ryzhik, Table of integrals, series and products. 1965: Academic Press.

65. Miller, M.K. and W. Guy, Numerical inversion of the Laplace transform by use of Jacobi polynomials. SIAM Journal on Numerical Analysis, 1966. 3(4): p. 624-635.

66. $\mathrm{Fu}, \mathrm{J}$., et al., Analytical solutions for multilayered composite cylinders with harmonic quadratic eigenstrain in arbitrary layers. Acta Mechanica, 2014. 225(2): p. 465-475. 